\title{
Central Sprouting and Functional Plasticity of Regenerated Primary Afferents
}

\author{
H. R. Koerber, ${ }^{2}$ K. Mirnics, ${ }^{1}$ P. B. Brown, ${ }^{2}$ and L. M. Mendell ${ }^{3}$ \\ ${ }^{1}$ Department of Neurobiology, University of Pittsburgh, Pittsburgh, Pennsylvania, 15261 2Department of Physiology, West \\ Virginia University, Morgantown, West Virginia, and ${ }^{3}$ Department of Neurobiology and Behavior, SUNY at Stony Brook, \\ Stony Brook, New York
}

\begin{abstract}
A combination of neuroanatomical and electrophysiological techniques was used to study the effects of peripheral axotomy and regeneration of primary afferents on their central projections in the spinal cord. Individual regenerated afferent fibers were impaled with HRP-filled electrodes in the dorsal columns of $\alpha$-chloralose-anesthetized cats and activated by current pulses delivered via the intracellular electrode. The resulting cord dorsum potentials (CDPs) were recorded at four rostrocaudal locations and HRP was iontophoretically injected into the fiber. Central distributions of boutons and CDPs were compared with peripheral receptor type to determine the accuracy of peripheral regeneration and the effects of central-peripheral mismatches. Reconstruction of the central projections of 13 individual afferents for which the adequate stimulus and CDPs had been recorded revealed many abnormalities. For example, unlike controls, four group I and II afferents with central projections typical of proprioceptors (concentrated in laminae V, VI, and VII) innervating either cutaneous or noncutaneous targets evoked measurable CDPs. Three other group II or A $\beta$ afferents innervating low-threshold mechanoreceptors with central terminations confined to the dorsal horn exhibited extensive collateralization in laminae $I$ and II in addition to large numbers of terminals in laminae III-IV. These fibers activated central networks whose adaptation behavior was identical to those evoked by high-threshold mechanoreceptive afferents in controls. These results suggest that primary afferents and their central connections are capable of significant modifications following axotomy and regeneration. In addition, the anatomical studies indicate some reorganization in the laminar distribution of boutons as well as in bouton size.
\end{abstract}

[Key words: spinal cord, plasticity, axotomy, regeneration, primary afferents, sprouting]

Peripheral nerve regeneration following transection has been studied extensively. It is known that following axotomy almost all myelinated fibers remain in the proximal stump and are

\footnotetext{
Received July 27, 1993; revised Nov. 3, 1993; accepted Nov. 24, 1993.

We thank Dr. Simon Watkins director of the Structural Biology Imaging Center at the University of Pittsburgh for the use of the OPTIMAS-based image analysis system and Sara Glickstein for excellent technical assistance. This work was supported by NIH Grants RO1 NS23275 (H.R.K.), RO1 NS16996 (L.M.M.), and PO1 NS14899 (L.M.M.).

Correspondence should be addressed to H. Richard Koerber, Department of Ncurobiology, University of Pittsburgh, School of Medicine, 808 Scaife Hall, Pittsburgh, PA 15261.

Copyright (C) 1994 Society for Neuroscience $0270-6474 / 94 / 143655-17 \$ 05.00 / 0$
}

available for regeneration (e.g., Hoffer et al., 1979). During regeneration approximately $75 \%$ of the fibcrs cross the ncuroma and reinnervate distal targets (Horch and Lisney, 1981). However, sensory fibers seldom return to the same locations they had previously innervated (Burgess and Horch, 1973; Horch, 1979), and in the case of axotomy of a large mixed nerve many reinnervate the wrong tissue type (Banks et al., 1984; Koerber et al., 1989). This ability of sensory fibers to reinnervate inappropriate peripheral targets has been confirmed in preparations in which both muscle and cutaneous nerves have been cut and rerouted into each others' distal stumps (Lewin and MacMahon, 1991a,b; Nishimura et al., 1993).

Peripheral axotomy and regeneration produce two major forms of sensory disorganization: (1) the somatotopic organization of inputs becomes scrambled and (2) submodality specificity is lost. Consequences of these abnormalities can be seen in the clinical descriptions of many symptoms following peripheral nerve injury. These symptoms vary greatly and range from inability to localize tactile stimuli to severe pain syndromes (e.g., allodynia) (Ford and Woodhall, 1938; Hawkins, 1948). Although some of these symptoms can last for years (most notably the pain syndromes: Lindblom and Verillo, 1979), other symptoms can show improvement with time (Moberg, 1962; Onne, 1962). Such improvement suggests that, in the absence of peripheral reorganization, some adaptive central reorganization occurs.

In order to study the possibility of spinal plasticity in response to faulty reinnervation it is first necessary to know when a fiber has reinnervated an inappropriate target. Afferent fibers are normally characterized by their responses to stimulation of their peripheral receptors, a method that cannot be used here. Therefore, another method of determining the preaxotomy identity of individual afferent fibers is necessary. The patterns of central terminations of primary afferents are characteristic for many afferent types (reviewed in Brown, 1981; Mense, 1990). In addition, recent studies have identified other properties of single primary afferents that are strongly correlated with the type of peripheral mechanoreceptor innervated in the periphery. These include somal membrane properties (Koerber et al., 1988), monosynaptic cord dorsum potentials (CDPs) (Koerber and Mendell, 1988), and frequency tuning of the afferent-driven spinal networks (Koerber and Mendell, 1988; Koerber ct al., 1991). Taken together, these parameters allow us to deduce with some certainty the type of peripheral receptor innervated by a given afferent before it was axotomized (Koerber et al., 1989).

The following experiments were designed to determine the specificity of regeneration following axotomy and self-cooptation of tibial and sural nerves and the effect of the appropriate- 
Table 1. Properties of regenerated afferent fibers

\begin{tabular}{|c|c|c|c|c|c|c|c|}
\hline \multirow{2}{*}{$\begin{array}{l}\text { Affer- } \\
\text { ent }\end{array}$} & \multirow{2}{*}{$\begin{array}{l}\text { Survival } \\
\text { time } \\
\text { (months) }\end{array}$} & \multirow{2}{*}{$\begin{array}{l}\mathrm{CV} \\
(\mathrm{m} / \mathrm{sec})\end{array}$} & \multirow{2}{*}{$\begin{array}{l}\text { Receptor type } \\
\text { after regeneration }\end{array}$} & \multirow{2}{*}{$\begin{array}{l}\text { Laminar location } \\
\text { of boutons }\end{array}$} & \multicolumn{2}{|c|}{ CDP properties } & \multirow{2}{*}{$\begin{array}{l}\text { Original } \\
\text { afferent type } \\
\text { by projection } \\
\text { pattern }\end{array}$} \\
\hline & & & & & Monosyn & $F / D$ & \\
\hline \multicolumn{8}{|c|}{ Proprioceptive afferents } \\
\hline$i$ & 10 & 88 & SA cut. LTMR & V, VI, VII, IX & No CDPs & & Ia spindle \\
\hline 2 & 13 & 96 & Spindle & V, VI, VII, IX & Yes & NT & Ia spindle \\
\hline 3 & 13.5 & 78 & SA type II & IV, V, VI, VII & Yes & NT & GTO \\
\hline 4 & 14 & 61 & SA type I & III, IV, V, VI & Yes & NR & Proprioceptor \\
\hline 5 & 10 & 62 & SA cut. LTMR & III, IV, V, VI & Yes & NT & Proprioceptor \\
\hline 6 & 12 & 65 & RA cut. LTMR & VI, VII, $(\mathrm{V})^{a}$ & No CDPs & & Proprioceptor \\
\hline \multicolumn{8}{|c|}{ Cutaneous afferents } \\
\hline 7 & 17 & 52 & RA pad & $\mathrm{III}, \mathrm{IV},\left(\mathrm{V}, \mathrm{II}_{\mathrm{i}}\right)$ & Yes & $-21 \%$ & Hair follicle \\
\hline 8 & 13.5 & 68 & Field & III, IV, (V) & Yes & $-27 \%$ & Hair follicle \\
\hline 9 & 9 & 60 & LTMR, insens. hair & III, IV & Yes & $-40 \%$ & SA type I \\
\hline 10 & 17.5 & 72 & SA, subcutaneous & III, IV, V, $\left(\mathrm{II}_{\mathrm{i}}\right)$ & Yes & $-7 \%$ & SA type I \\
\hline \multicolumn{8}{|c|}{ Sprouted cutaneous afferents } \\
\hline 11 & 14 & 62 & RA cut. LTMR & I, II, IV, V & Yes & NT & cutaneous \\
\hline 12 & 17 & 68 & SA, cutaneous & I, II, III, IV, V & Yes & $+54 \%$ & cutaneous \\
\hline 13 & 14 & 69 & SA, subcutaneous & I, II, III, IV, (V) & Yes & $+10 \%$ & cutaneous \\
\hline
\end{tabular}

NT, not tested; NR, no response; F/D, facilitation/depression; GTO, Golgi tendon organ.

"Laminae in parentheses denotes that fewer than $1 \%$ of boutons were found in these laminae.

ness of reinnervated target on the central anatomy and function of individual afferent fibers.

Preliminary results have been published elsewhere (Koerber et al., 1989, 1992).

\section{Materials and Methods}

Axotomies. Adult cats unselected as to sex were anesthetized with a mixture of ketamine (Ketaset, $30 \mathrm{mg} / \mathrm{kg}$ ) and xylazine (Rompun, 1.5 $\mathrm{mg} / \mathrm{kg}$ ). Under aseptic conditions the tibial and sural nerves were exposed in the popliteal fossa. The two nerves were transected and the cut ends were anastomosed using epineural sutures (7.0-9.0 ophthalmic). The transection sites and self unions of the two nerves were usually $1-2 \mathrm{~cm}$ apart in the fossa with the sural nerve site always more proximal. The wound was sutured in layers and treated with topical antiseptic ointment. Each animal was given an intramuscular injection of antibiotic (amoxicillin, $50 \mathrm{mg}$ ) and recovered without incident.

Recording and iontophoresis. The animals were initially anesthetized with an intramuscular injection of a mixture of ketamine $(20 \mathrm{mg} / \mathrm{kg})$ and xylazine $(1.0 \mathrm{mg} / \mathrm{kg})$. An external jugular vein, common carotid artery, and the trachea were cannulated in order to infuse drugs, monitor blood pressure, and provide ventilation. Following the cannulations the anesthetic was switched to $\alpha$-chloralose with an initial dose of $60 \mathrm{mg}$ and followed by $30 \mathrm{mg}$ supplements to a total of $70 \mathrm{mg} / \mathrm{kg}$. Animals were placed in a rigid frame and a dorsal laminectomy was performed to expose the lumbosacral enlargement and dorsal root ganglia. The L6-S1 spinal segments were freed from the dura by cutting the dentate ligaments and a smooth Plexiglas platform was placed under the L7 segment. The platform was elevated slightly in order to stabilize the cord to facilitate stable intraaxonal recordings.

The left hindlimb was fixed using a bone pin inserted in the medial surface of the tibia. The tibial, peroneal, and sural nerves were exposed proximal to the transection site and placed on platinum bipolar stimulating electrodes either individually or more usually, due to space limitations and/or difficulty with separation of the sural and tibial nerves as a consequence of the initial surgery, with sural and tibial on one pair and the peroneal on another. The nerves and electrodes were isolated from each other using Parafilm. This arrangement allowed identification of fibers as regenerated or intact. Following placement of the stimulating electrodes four bipolar platinum recording electrodes were positioned with the active leads centered over the dorsal horn and the indifferents placed in the surrounding muscle. The rostrocaudal locations were determined as in previous publications (Koerber and Mendell, 1988).
While stimulating the tibial and sural nerves at $2 \mathrm{~Hz}$ a microelectrode containing HRP ( $15 \%$ in Tris buffer; $\mathrm{pH} 7.6$ ) was advanced through the dorsal columns in search of responding fibers. Once a regenerated afferent fiber was suitably impaled (action potential $\geq 60 \mathrm{mV}$; resting membrane potential $\leq-60 \mathrm{mV}$ ), its conduction latency to nerve stimulation was recorded, its peripheral receptive field was located, and receptor properties were characterized using hand-held probes. Afferents were first identified as cutaneous or noncutaneous. Next they were broadly classified as rapidly or slowly adapting. Finally, they were further characterized as a specific afferent type (e.g., slowly adapting type I) when possible. The afferent was then stimulated with positive current pulses lasting $100 \mu \mathrm{sec}$ delivered through the intracellular electrode, with each current pulse evoking one action potential in the afferent fiber. Stimulus presentations included one shock every $56 \mathrm{msec}(18 \mathrm{~Hz})$ and pairs of shocks every $2 \mathrm{sec}$ (intershock interval $=50 \mathrm{msec}$ ). The resulting cord dorsum potentials (CDPs) were averaged and stored for later analysis. The individual fibers (one per experiment) were then filled with HRP by iontophoretic injection (10-20 nA positive current pulses; $75 \%$ duty cycle). The primary criterion for selection was the ability to characterize unambiguously the peripheral response properties. An equally important criterion was an adequate membrane potential and a stable penetration. Following the injection procedure the microelectrode was removed and the preparation was maintained for another $6-12 \mathrm{hr}$ to permit diffusion of HRP into afferent fiber terminals in the dorsal horn. During this time each preparation was used to acquire physiological data (to be presented in a subsequent report) but no microelectrodes were reintroduced into the spinal cord until marker electrodes were inserted just before perfusion. These electrodes were placed in the midline at the corresponding rostrocaudal location of one or more of the surface recording electrodes, and the body of the electrode was cut off leaving the visible shank embedded in the cord. The distance between cord dorsum electrodes was recorded, $1000 \mathrm{U}$ of heparin administered and the animal was perfused with $1000 \mathrm{ml}$ of saline $\left(37^{\circ} \mathrm{C}\right)$ followed by $3000 \mathrm{ml}$ of $2 \%$ glutaraldehyde and $0.5 \%$ paraformaldehyde in phosphate buffer ( $\mathrm{pH} \mathrm{7.4)}$ at $4^{\circ} \mathrm{C}$. A $20-24 \mathrm{~mm}$ block of spinal cord spanning the length of the electrode array was removed and its length and the locations of marker electrodes were recorded. The tissue was stored for $8-12 \mathrm{hr}$ in a $30 \%$ sucrose-phosphate buffer solution. The block was sectioned on a freezing microtome taking $100 \mu \mathrm{m}$ sagittal sections from the entire length of the block. Sections were processed using the cobalt-enhanced diaminobenzidine method (Adams, 1977) and counterstained with neutral red.

The central projections of the individual afferents were reconstructed and the rostrocaudal and laminar distribution of boutons determined. 
The rostrocaudal distribution was detcrmined by counting all stained boutons in $250 \mu \mathrm{m}$ rostrocaudal bins. Individual afferent bouton counts were then summed across all sections containing stained material. These distributions could then be compared to the distribution of CDPs recorded at the four surface sites (see Koerber et al., 1990, for details).

Bouton areas were measured using a $100 \times$ oil objective, a video camera, a frame grabber card, and a semiautomated software package (OPTIMAS). Once a field of view had been selected, the operator adjusted the contrast threshold such that all stained elements were detected in their entirety. Each selected stained profile was then evaluated with regard to its closeness of fit to an ellipse. The level of error was adjusted to permit routine distinction between boutons and other stained elements. In order to make sure that only boutons were selected for measurement the error threshold was left relatively low so that in a given ficld of vicw not all boutons would be sclcctcd but only boutons were selected. The boutons that were not selected by these methods were en passant boutons on relatively large diameter collateral fibers. Once this level was determined it was used for all subsequent measurements. It was necessary for the operator to readjust the contrast threshold from section to section because of differences in the density of background and counterstaining. In addition the operator determined the accuracy of bouton selection for each field of view before the measurements were made. Due to the subjective decisions required, all material was coded so that the operator did not know which sections contained control or experimental material, and the order in which animals were processed was independent of afferent classification (e.g., receptor type, survival time).

All control data used for comparisons with the results of the present study were either from previously published data (Kocrber ct al., 1990) or based on reanalysis (i.e., bouton measurements) of those data.

\section{Results}

Since these fibers had previously been transected and had reinnervated a receptor in the periphery, classification of their original type by receptor properties would not be appropriate (see introductory remarks). However, the central projection patterns of the fibers in this study could easily be divided in to two groups: those with projections confined to the dorsal horn, and those projecting into the intermediate gray and the ventral horn. Therefore, fibers were initially divided in to originally cutaneous and proprioceptive groups by the locations of their central terminations in the spinal cord. This classification was based on the assumption that individual afferents did not completely rearrange their central projections (i.e., from proprioceptive projections primarily in VI and VII, to cutaneous projections primarily in III, IV, and V).

A total of 13 afferents was injected in 13 animals 9-17.5 months following axotomy. Although the staining did not always label the full rostrocaudal extent of every afferent, each injection did label only one afferent fiber and enough of the central projection was recovered to allow a qualitative evaluation of the pattern of its central terminations. Of the 13 afferent fibers stained in this study, six supported terminals located predominantly in laminae V, VI, and VII and were judged to have been proprioceptive originally. Of these six, three (afferents 13 , Table 1) had conduction velocities in the group I range (78, 88 , and $96 \mathrm{~m} / \mathrm{sec}$ ). Two of the three (afferents 1, 2, Table 1) also had collaterals extending into lamina IX $(\mathrm{CV}=88$ and 96 $\mathrm{m} / \mathrm{sec}$ ), and so these were further classified as primary spindle afferents. The remaining three fibers (afferents 4-6, Table 1) had conduction velocities in the group II range $(61,62$, and 65 $\mathrm{m} / \mathrm{sec}$ ).

The other seven afferents (afferents 7-13, Table 1) had conduction velocities in the group II or $A \beta$ range and maintained extensive collaterals in laminae III-IV and so were judged to have been cutaneous afferents originally. Three of these afferents (afferents 11-13, Table 1), with conduction velocities ranging

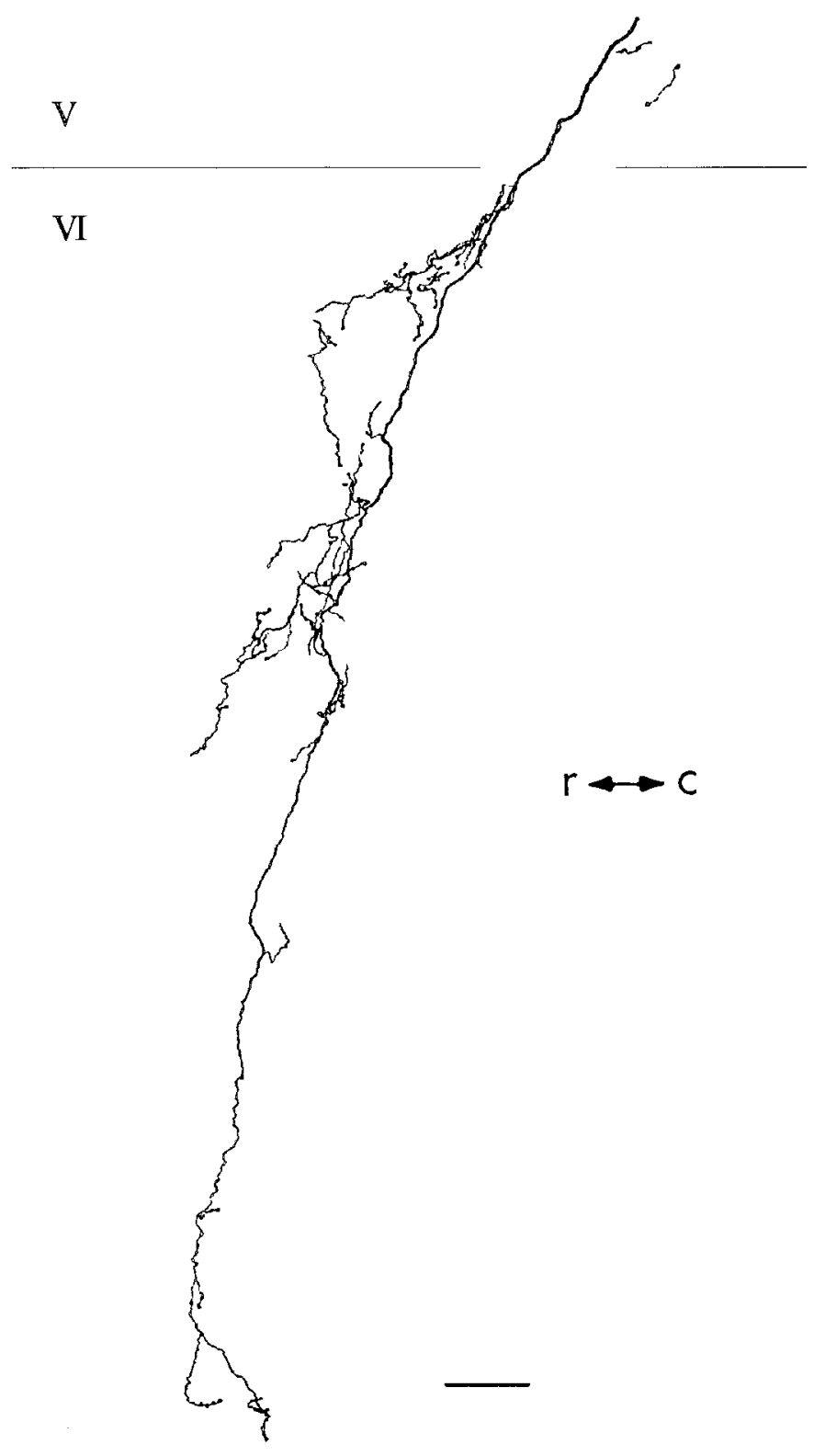

Figure 1. Camera lucida drawing of a sing : collateral laken from sagittal section. This group I fiber had a conduction velocity of $88 \mathrm{~m} / \mathrm{sec}$ and its projections extended into lamina IX (ventralmost part of projection shown). This fiber was judged to have innervated a muscle spindle before axotomy. Note the lack of boutons dorsal to lamina VI. $r$, rostral; $c$, caudal. Scale bar, $100 \mu \mathrm{m}$.

from 62 to $68 \mathrm{~m} / \mathrm{sec}$, also supported longitudinally oriented collaterals in laminae I and II. The remaining four afferent fibers (afferents 7-10, Table 1) also had conduction velocities in the A $\beta$ range $(52-72 \mathrm{~m} / \mathrm{sec})$. All seven fibers returning to the skin had small and concise receptive fields. Those reinnervating noncutaneous structures could not always be adequately examined but no obvious split receptive fields were encountered in this population.

\section{Originally proprioceptive fibers}

Of the six afferents with central projections typical of proprioceptive fibers, five reinnervated the skin (afferents 1, 3-6, Table 1) while the remaining fiber (afferent 2, Table 1) apparently reinnervated muscle and exhibited a stretch-evoked response. 


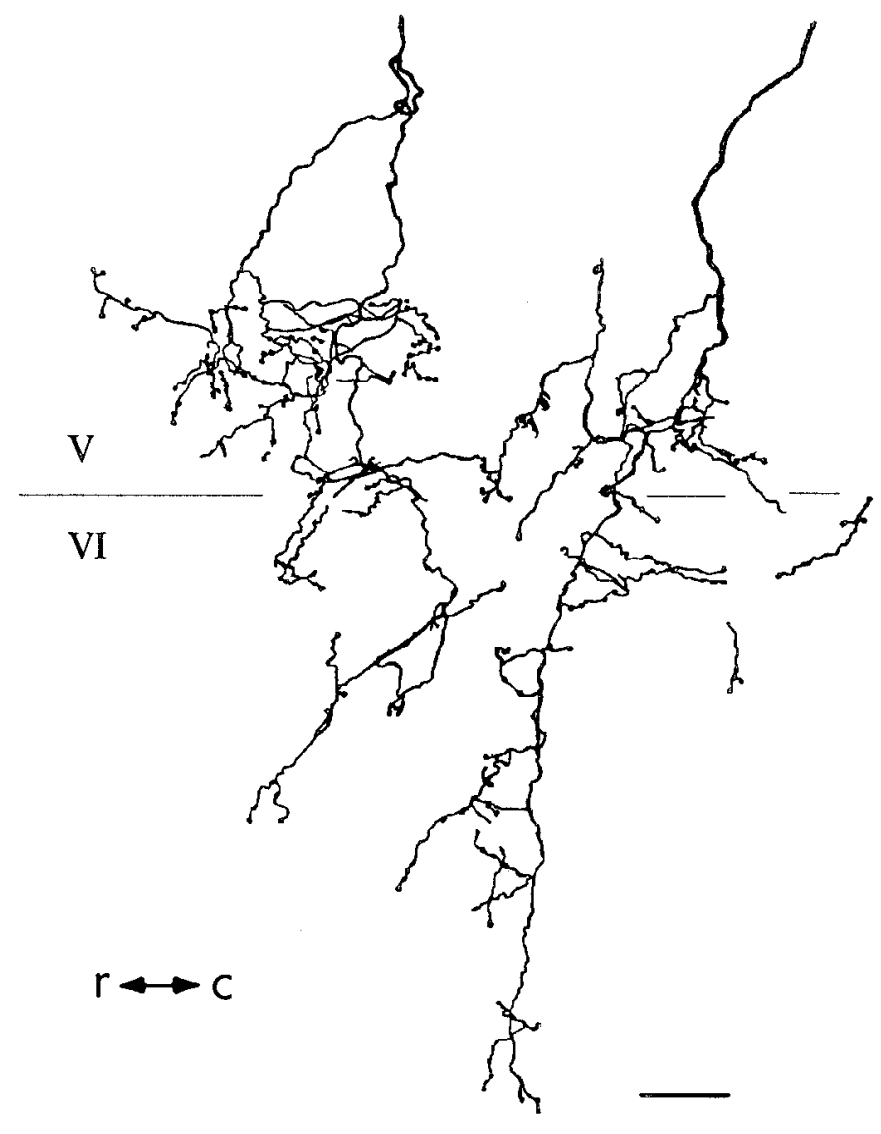

Figure 2. Camera lucida drawing of two adjacent collaterals viewed in the sagittal plane. This group I fiber had a conduction velocity of 96 $\mathrm{m} / \mathrm{sec}$ and supported projections in lamina IX (most ventral part of projection). This fiber was judged to have innervated a muscle spindle before axotomy. Note the large numbers of boutons dorsal to lamina VI. $r$, rostral; $c$, caudal. Scale bar, $100 \mu \mathrm{m}$.

The previously proprioceptive fibers reinnervating the skin included the three group II and two of the group I afferents. These afferents were not uniform in their responses to skin stimulation, with one characterized as rapidly adapting and four as slowly adapting.

The three group I fibers had very different properties. Of the two that had collaterals in lamina IX, one (Fig. 1) had a conduction velocity of $88 \mathrm{~m} / \mathrm{sec}$ and innervated glabrous skin (low threshold, slowly adapting). This fiber (afferent 1, Table 1) did not evoke measurable CDPs in response to $18 \mathrm{~Hz}$ stimulation. The other one (afferent 2, Table 1), shown in Figure 2, had a conduction velocity of $96 \mathrm{~m} / \mathrm{sec}$, reinnervated muscle, exhibited a stretch-evoked response and evoked measurable CDPs on all four electrodes (Fig. $3 A$ ). The apparent reason for the difference in CDP production was that the former afferent had fewer than $1 \%$ of its boutons in laminae IV-V whereas the latter afferent had $51 \%$ of its boutons in IV and V. It is important to note that in control animals proprioceptive afferents do not produce CDPs (Koerber and Mendell, 1988), and so CDPs represent a modification of their central action.

The third group I fiber (afferent 3, Table 1) reinnervated hairy skin and exhibited some spontaneous activity. This fiber was characterized as slowly adapting and discharged very regularly to low-intensity stimulation of the skin. Figure 4 displays the reconstruction of three representative collaterals of this fiber.
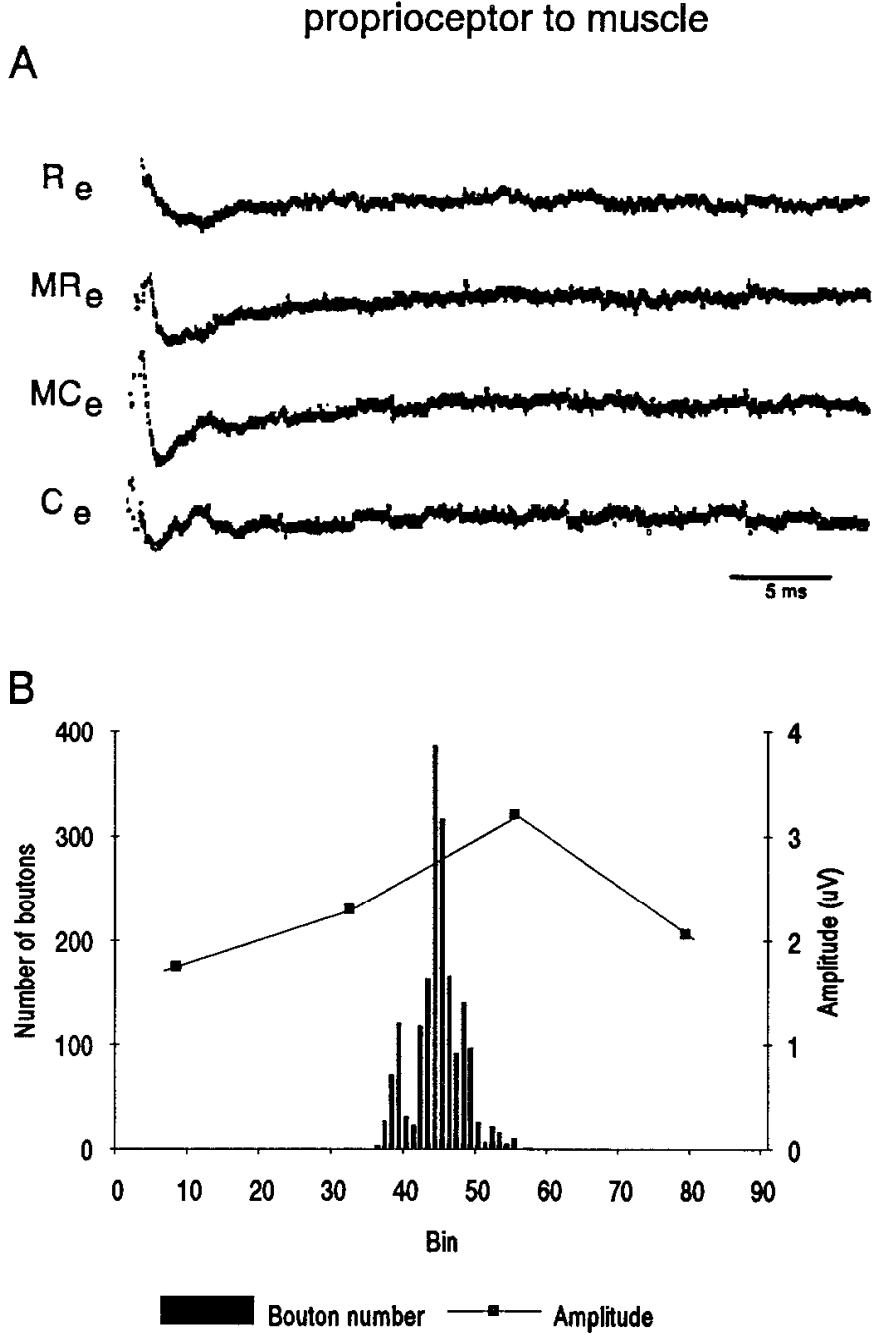

Figure 3. A, CDPs recorded at four different sites in response to 18 $\mathrm{Hz}$ stimulation of the group I afferent fiber depicted in Figure 2. $B$, Rostrocaudal distribution of boutons $(250 \mu \mathrm{m}$ bins) and the amplitudes of the CDPs recorded at the four sites plotted in register. $R_{e}$, rostral electrode; $M R_{c}$, mid-rostral electrode; $M C_{e}$, mid-caudal electrode; $C$ caudal electrode.

Although this afferent exhibited its highest bouton density in lamina VI, there were significant numbers of boutons in lamina $\mathrm{V}$ and $\mathrm{a}$ few in laminae IV and VII. For group I fibers this pattern of termination is most similar to that reported for group Ib fibers (Brown, 1981). Stimulation of this fiber evoked CDPs of relatively constant amplitude on all four electrodes (Fig. 5A). This was a consistent finding for the CDPs evoked by stimulation of afferents that were apparently proprioceptors before axotomy (see below). We have plotted the rostrocaudal distribution of boutons in laminae IV-VII along with the amplitudes of the CDPs recorded at the four surface locations (Figs. $3 B$, $5 B$ ). The agreement betwecn the bouton distributions and amplitude profiles for these two afferents does not appear to be as close as that seen for intact cutaneous afferents (Koerber et al., 1990) or some of the regenerated cutaneous afferents in the present study (see below). However, since only two of the proprioceptive afferents were stained well enough to warrant counting boutons, statistical analysis was not appropriate.

The remaining three proprioceptive fibers were group II and each reinnervated the skin. Two of them were classified as slowly 


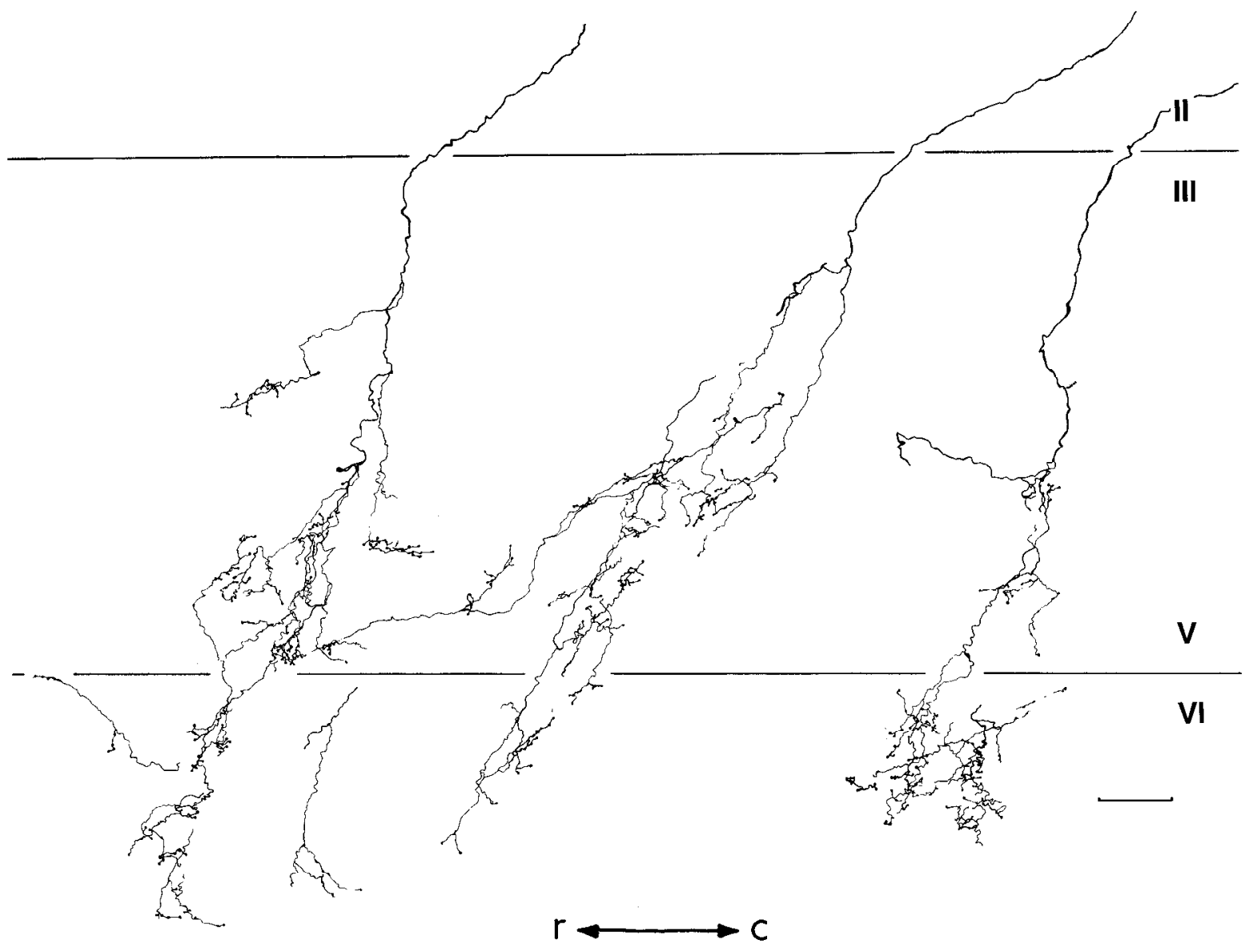

Figure 4. Camera lucida drawing of three representative collaterals of a third group I fiber $(\mathrm{CV}=78 \mathrm{~m} / \mathrm{sec})$; reconstruction from several sagittal sections. Note the presence of collateral fibers and boutons in laminae IV and V. $r$, rostral; $c$, caudal. Scale bar, $100 \mu \mathrm{m}$.

adapting low-threshold mechanoreceptors (LTMRs) (afferents 4,5 , Table 1 ) and the third was rapidly adapting (afferent 6 , Table 1). Of these fibers, two evoked CDPs (afferents 4,5 ) in response to intracellular stimulation while the third did not elicit a measurable response. Once again the major difference between the central projections of those producing measurable CDPs and the one that did not is that the former supported more dorsally located boutons. Two such afferents are compared in Figures 6 and 7 , which are camera lucida drawings of representative portions of the fibers' projections in the sagittal plane. The afferent depicted in Figure 6 had a conduction velocity of $61 \mathrm{~m} / \mathrm{sec}$, had reinnervated the glabrous skin of toe 5 , and exhibited a slowly adapting response to low-intensity stimulation (afferent 4 , Table 1). This afferent supported numerous collaterals and boutons in lamina $\mathrm{V}$ and some in IV and evoked measurable CDPs in response to $18 \mathrm{~Hz}$ stimulation. In contrast the second fiber, which reinnervated hairy skin and exhibited a low-threshold rapidly adapting response (Fig. 7), supported only a few boutons dorsal to lamina VI and did not evoke measurable CDPs (afferent 6). Although these fibers had central termination patterns that were clearly those of proprioceptors, more specific classification (e.g., secondary spindle) was not possible.

The CDPs evoked by $18 \mathrm{~Hz}$ stimulation of both group I and II regenerated proprioceptive fibers had latencies to onset $(<1$ $\mathrm{msec}$ ) and rise times $(0.60-0.75 \mathrm{msec})$ consistent with monosynaptic linkage reported previously for CDPs evoked by $\mathrm{cu}-$ taneous afferents (Koerber and Mendell, 1988). However, the amplitudes were relatively small $(2.03 \pm 0.44 \mu \mathrm{V})$ compared to the regenerated cutaneous fibers (see below).

\section{Originally cutaneous fibers}

Of the seven fibers classified as being previously cutaneous, five reinnervated skin and two had returned to subcutaneous tissue. As stated above these fibers could be further divided into those with central projections indicative of low-threshold cutaneous mechanoreceptors (LTMRs) in the control population (classified as cutaneous afferents) and those that supported additional collaterals in laminae I and II (classified as sprouted cutaneous afferents).

Cutaneous afferents. The four afferent fibers classified as cutaneous LTMRs supported central terminations that upon microscopic examination were indistinguishable from those observed in controls. Two of these fibers supported central projections that ended in characteristic flame-shaped arbors in lamina III. The central termination pattern of one of these, viewed in the sagittal plane (afferent 8 , Table 1), is depicted in Figure $8 A$. The main collaterals of these afferents remained in the dorsal aspect of the dorsal columns over the length which was stained. These anatomical features indicate that these fibers were most likely to have innervated hair follicles before axotomy (Koerber et al., 1990). Both of these fibers were also classified as rapidly adapting following regeneration. One fiber (afferent 8 , Table 1) exhibited a rapidly adapting response to stimulation of hairy skin (but not to deflection of hairs alone) and was 


\section{proprioceptor to skin}

A

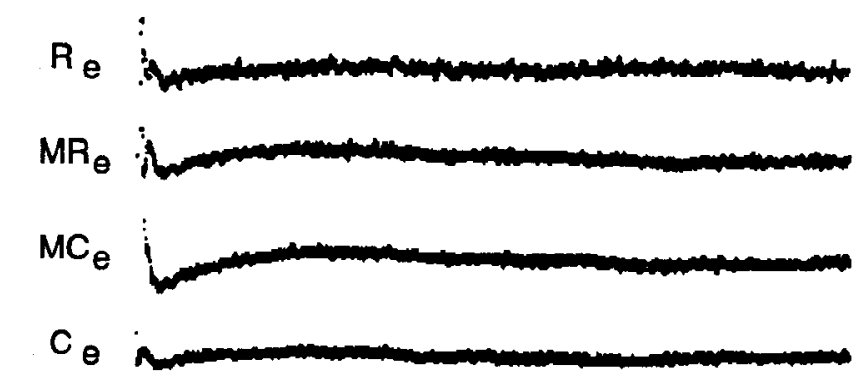

$5 \mathrm{~ms}$

B

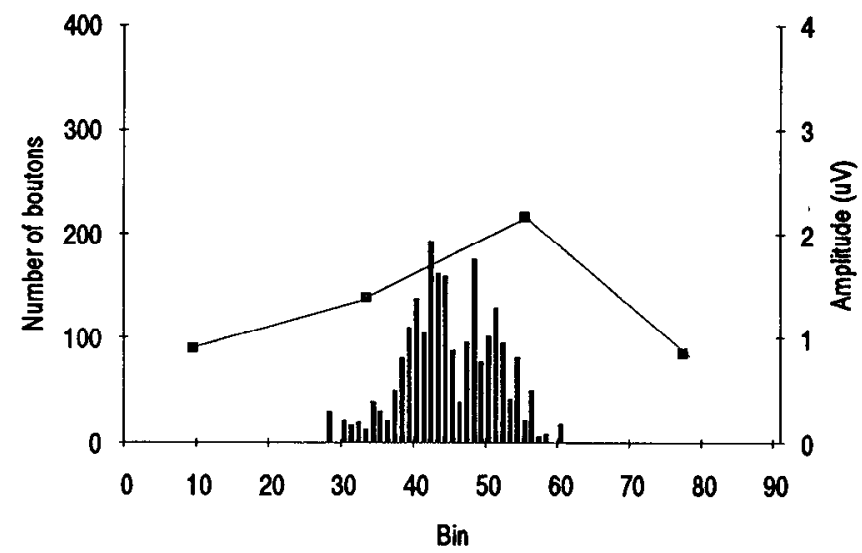

Bouton number $\longrightarrow$ Amplitude

Figure 5. $A$, CDPs recorded in response to $18 \mathrm{~Hz}$ stimulation of the group I afferent fiber depicted in Figure 4. $B$, Rostrocaudal distribution of boutons ( $250 \mu \mathrm{m}$ bins) and the amplitudes of the CDPs recorded at the four sites plotted in register.

classified as a field receptor, while the other (afferent 7, Table 1) innervated glabrous skin and was classified as an RA pad. The other two cutaneous afferents had central terminals that more closely resembled those of slowly adapting afferents (Fig. $8 B$ ) in controls (Brown, 1981; Koerber et al., 1990). Of these, one responded in a slowly adapting fashion to stimulation of subcutaneous tissue (afferent 10, Table 1), while the other was found to have reinnervated hair follicles (afferent 9, Table 1). This fiber responded sluggishly to manual deflection of hairs but was clearly rapidly adapting.

All four of these fibers evoked measurable CDPs in response to intracellular stimulation. CDPs evoked in response to $18 \mathrm{~Hz}$ stimulation had a clear monosynaptic component which could be easily resolved (Figs. $9 A, C, E ; 10 A$ ). These monosynaptic CDPs had rise times $(<1 \mathrm{msec})$ and amplitudes $(3.3-9.2 \mu \mathrm{V})$ that were within normal limits for cutaneous afferent fibers (Koerber and Mendell, 1988; Koerber et al:, 1990). For three of the four fibers (afferents 7, 8, 10) the CDPs (Fig. 9A,C,E) were indistinguishable in configuration from those observed in the control population. For example, the CDPs shown in Figure $9 C$ have a "scooped out" appearance similar to that observed in response to stimulation of hair follicle afferents in controls (see Fig. 6 in Koerber and Mendell, 1988). Note that as in the earlier study there was a clear break after the monosynaptic peak that could be easily distinguished.

These same fibers were activated with pairs of shocks at 50 msec intervals in a conditioning-testing paradigm that was repeated every $2 \mathrm{sec}$. The resulting CDPs, averaged in register, were again very normal in appearance (Fig. $9 B, D, F$ ). The conditioning stimulus in each RA afferent fiber resulted in depression of the response to the test stimulus $(21 \%$ and $27 \%)$ that is similar to the depression for RA afferents in controls (Koerber and Mendell, 1988). As in the control population the facilitation/depression behavior was determined for the location where the largest CDPs were recorded (Koerber and Mendell, 1988). In the control preparations the facilitation/depression behavior was consistent for single-fiber CDPs recorded at the four sites (Koerber and Mendell, 1988). However, this was not the case for the afferent in Figure 9B, where the test CDP in the bottom trace exhibited facilitation whereas in the other traces the test CDP is depressed compared to the conditioning CDP. Although this is only a single case, it may be indicative of functional plasticity in the dorsal horn after peripheral regeneration.

One of the anatomically described SA fibers (afferent 10, Table 1) evoked CDPs that were similar in appearance to those of SA afferents in controls (Fig. $9 F$ ). Specifically, the response to the second (or test) shock was relatively unaffected by the conditioning shock ( $7 \%$ depression; Fig. $9 F$ ). The remaining cutaneous afferent had central projections that were identical to those seen for SA fibers in controls (Fig. 8B; compare with Fig. $2 a$ in Koerber et al., 1990). High-frequency stimulation of this fiber evoked CDPs that consisted of a monosynaptic component followed by a polysynaptic potential with a slow rise time (Fig. $10 A$ ). This type of response is uncharacteristic of SA fibers in controls, which evoke no measurable polysynaptic component in response to $18 \mathrm{~Hz}$ stimulation (Koerber and Mendell, 1988). This SA fiber also exhibited an unusual response to the conditioning-testing pairs of pulses by showing a substantial depression (40\%), a level characteristic of RA fibers in controls. Although there is an apparent mismatch between the anatomy and the facilitation/depression response, it should be pointed out that the fiber did reinnervate hair follicles and responded in a rapidly adapting fashion. Thus, in this case there was a good correlation between reinnervated receptor function and the central response.

One final abnormal aspect of the CDPs evoked by this fiber was that the polysynaptic response was largest at a recording site other than where the largest monosynaptic CDP had been recorded. This result is depicted graphically in Figure $10 B$. Note that the two amplitude profiles are out of register. Such amplitude profiles were always in register in controls (Koerber and Mendell, 1988).

Sprouted cutaneous afferents. All three afferents characterized anatomically as sprouted cutaneous afferents were found to have reinnervated peripheral receptors that responded to innocuous mechanical stimulation of either the skin $(n=2)$ or subcutaneous tissue $(n=1)$. Examination of the central terminations of these $A \beta$-fibers revealed projections that were similar to the projections for $A \beta$ LTMRs with the exception of the collaterals and boutons in laminae $\mathrm{I}$ and $\mathrm{II}_{0}$. Examples of these projections can be seen in Figure $11, A$ and $B$. Figure $11 A$ is a camera lucida drawing of a selected portion of the central terminations of one of these fibers (afferent 13, Table 1). It is clear that the bulk of 


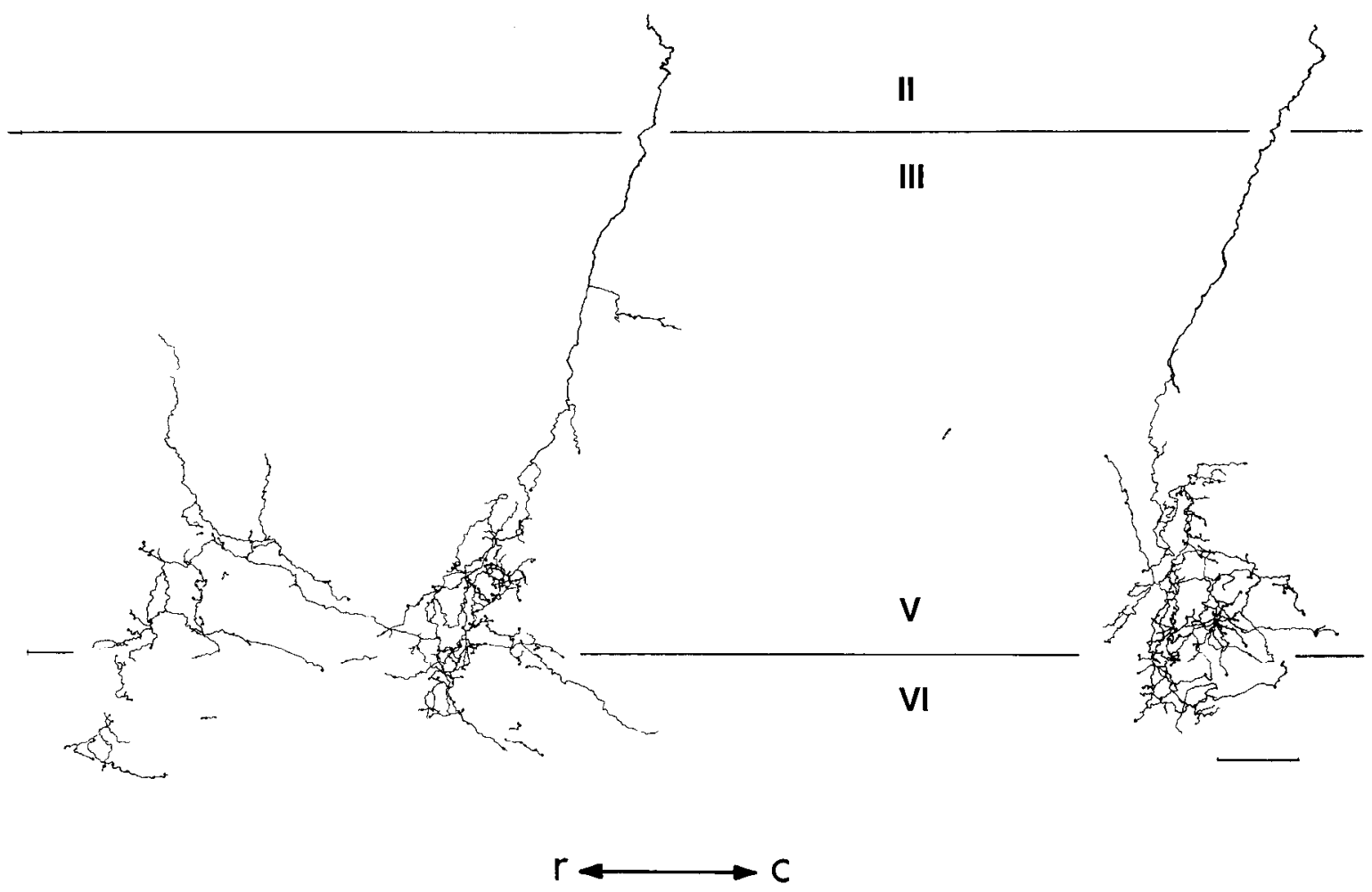

Figure 6. Camera lucida reconstruction of representative collaterals of a group II fiber judged to have been a proprioceptive afferent fiber before axotomy. Collaterals viewed in sagittal section. Note boutons in lamina V. $r$, rostral; $c$, caudal. Scale bar, $100 \mu \mathrm{m}$.

collaterals and boutons supported by this fiber are located in laminae III and IV. However, significant numbers of longitudinally oriented fibers are also supported in the more superficial laminae. This is a hybrid of the normal projection patterns of LTMRs and nociceptors and we assume that this had been a cutaneous LTMR afferent that had sprouted terminals into superficial laminae of the dorsal horn (Woolf et al., 1992; Shortland and Woolf, 1993). A lower-power view of the entire stained collateralization of a second such fiber (afferent 11, Table 1) can be seen in Figure $11 \mathrm{~B}$. Once again this fiber supported a hybrid central termination pattern of longitudinally oriented collaterals in the superficial laminae and transversely oriented collaterals penetrating the nucleus proprius.

Intracellular stimulation also revealed interesting differences between these $\mathrm{A} \beta$-fibers and those described earlier. In response to $18 \mathrm{~Hz}$ stimulation these fibers evoked CDPs (Fig. 12A,C,D) with slow rise times (1.4-2.4 msec) that did not have obvious short-latency peaks indicative of a monosynaptic component, as seen for the fibers depicted in Figures 9 and 10. Although these rise times are slower than those observed for $\mathrm{A} \beta$ LTMRs in controls, they are similar to those recorded for HTMRs (Koerber and Mendell, 1988). The paired shock stimulus paradigm was delivered to two of these fibers. In both cases the test CDP response exhibited facilitation. In one case this facilitation was $54 \%$, and in the other it was $10 \%$ (Fig. $12 B$ ). Once again these results are consistent for $\mathrm{A} \beta$ HTMRs in the control population (Koerber and Mendell, 1988). Thus both anatomically and physiologically these large myelinated fibers exhibit characteristics of nociceptors. Due to their high conduction velocities $(62,68$, and $69 \mathrm{~m} / \mathrm{sec}$ ) and numbers of collaterals and boutons in laminae III and IV, it appears that these fibers had previously been cutaneous LTMRs that following axotomy and regeneration had extended projections into the superficial laminae. In addition, these projections appear to have made functional connections with dorsal horn cells in these laminae as the CDPs elicited by these fibers had the appearance of nociceptor-evoked CDPs in controls.

\section{Comparison of $C D P$ s from regenerated proprioceptive and cutaneous afferents}

On visual inspection the CDPs evoked by $18 \mathrm{~Hz}$ stimulation of regenerated proprioceptive fibers differed from those recorded in response to stimulation of regenerated cutaneous afferents. Specifically, the proprioceptive fiber-evoked CDPs exhibited relatively small differences in amplitude at the four different sites when compared to the regenerated cutaneous fiber-evoked CDPs. In order to quantify this observation the standard deviation of the amplitude distributions was calculated (Koerber et al., 1990) for these afferents and the regenerated cutaneous afferents. The SD of the distribution was significantly larger for the regenerated proprioceptive afferents $(5.83+0.33 \mathrm{~mm})$ than for the cutaneous afferents $(4.69 \pm 0.25 \mathrm{~mm} ; t=-2.73 ; p=$ 0.02 ). This larger dispersion for the proprioceptive fibers equates to a flatter amplitude distribution. Another difference between regenerated proprioceptors and cutaneous fibers was that when one of these proprioceptive fibers was stimulated using the paired shock paradigm there was no sign of polysynaptic components in the CDPs that are usually present in response to low-frequency stimulation of cutaneous afferents (Koerber and Mendell, 1988; Koerber et al., 1991; also see Fig. 9).

\section{Correlation of $C D P S$ and bouton distributions}

A total of seven fibers (five cutaneous and two proprioceptive) were selected for bouton counts. These fibers were chosen be- 


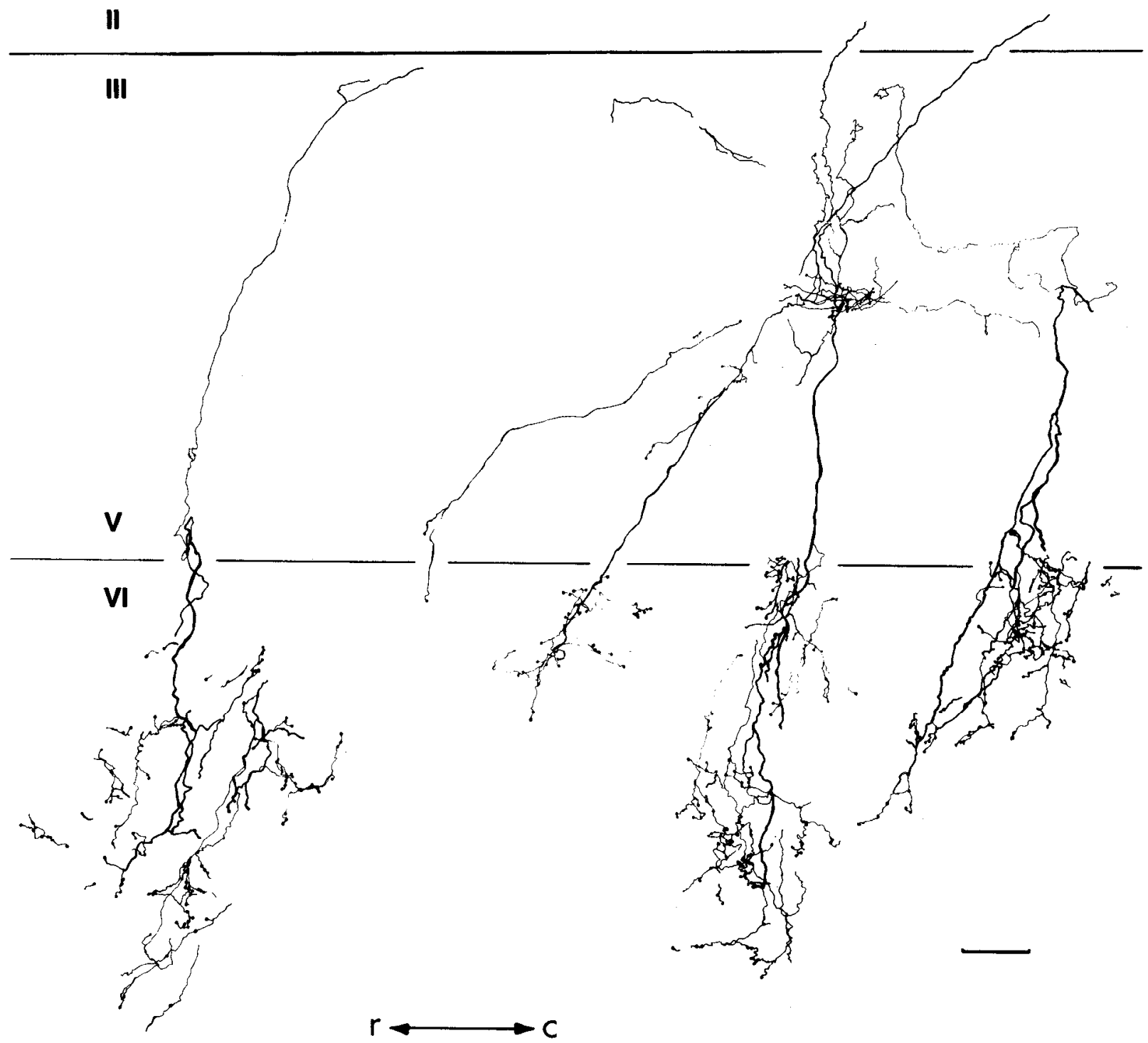

Figure 7. Camera lucida drawing of selected collaterals of a second group II proprioceptive afferent fiber $(\mathrm{CV}=65 \mathrm{~m} / \mathrm{sec})$. Note that most boutons are ventral to lamina V. $r$, rostral; $c$, caudal. Scale bar, $100 \mu \mathrm{m}$.

cause the intensity of staining was judged to be equivalent to that for fibers used in control studies. Only the five originally cutaneous fibers were used for comparisons with controls since the latter were restricted to cutaneous fiber projections (Koerber et al., 1990). The rostrocaudal distribution of boutons in the dorsal horn was then plotted along with the amplitude profile for each fiber and the correlation between the locations of the boutons and the amplitudes of observed CDPs were directly compared with amplitudes predicted on the basis of bouton distributions. The match between these two measurements was very good for several of the afferents (Fig. 13D,E; afferents 13, 10, Table 1) and was similar to that seen in the control study (Koerber et al., 1990). That is, the peak of the bouton distribution matched closely the peak of the amplitude profile. However, for some of the fibers the correlation was smaller than in controls (Fig. 13A,B; afferents 8, 9, Table 1), and in one case the peak of the CDP amplitude profile was displaced relative to the peak of the bouton distribution (Fig. 13A), which was never seen in controls.

In the earlier study we were able to construct predicted CDP amplitude profiles using the bouton distributions, an assumption concerning the decrement of the synaptic potential and a calculated amplitude coefficient " $a$ " (Koerber et al., 1990). In normal preparations the observed and predicted CDP amplitude profiles were very much in agreement. Predicted CDP amplitude profiles were also constructed for selected regenerated cutaneous fibers. Examples of the agreement between predicted and observed can be seen for the five afferents in Figure 13. The mismatches between the bouton distributions and amplitude profiles in the rostrocaudal axis are quite obvious for some of the afferents. For example, for the fiber depicted in Figure $13 \mathrm{~A}$, the location at which the largest amplitude CDP was recorded was different from that predicted using these calculations. Another component of the mismatch that becomes apparent in these plots is the differences in amplitudes at the different locations. The differences between predicted and observed potentials at the individual locations appeared to be much larger for the regenerated fibers than the control population (Koerber et al., 1990).

In order to determine if these differences were significant we 
II

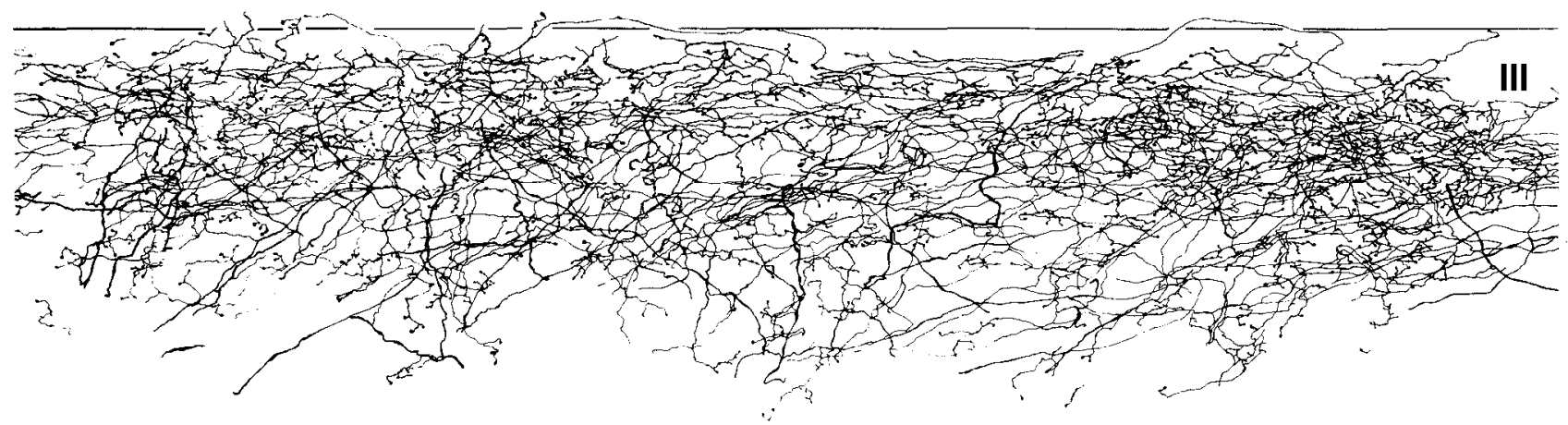

B
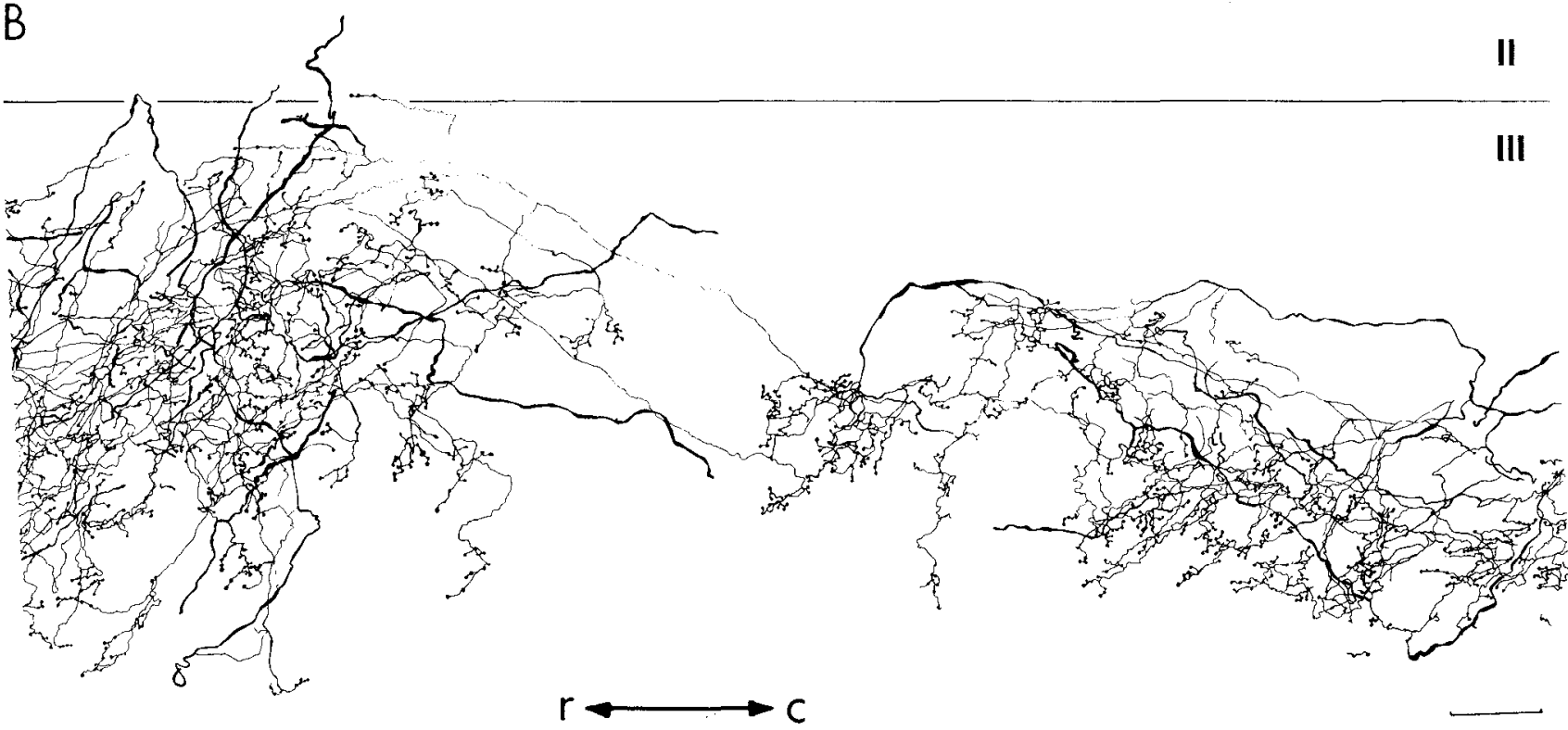

Figure 8. A, Camera lucida drawing of a portion of the central termination of an $A \beta$-fiber $(\mathrm{CV}=68 \mathrm{~m} / \mathrm{sec})$ judged to have been a cutaneous afferent fiber before axotomy. The CDPs evoked by stimulation of this afferent can be seen in Figure $9, A$ and $B$. $B$, Camera lucida drawing of a second $\mathrm{A} \beta$-fiber $(\mathrm{CV}=60 \mathrm{~m} / \mathrm{sec})$ judged to have innervated the skin before axotomy. The CDPs evoked by stimulation of this fiber are presented in Figure 10A. Note the distinct difference between the projection patterns of these cutaneous fibers and the proprioceptive fibers shown in Figures 6 and 7. $r$, rostral; $c$, caudal. Scale bar, $100 \mu \mathrm{m}$.

have quantificd the differences between the predicted and observed amplitudes by determining the percentage error. This was calculated as the absolute value of 1 - (predicted amplitude/ observed amplitude) $\times 100$. As before, in order to minimize any influence of the quality of staining we have made the comparisons only at the two recording sites at which the largest CDPs were recorded. The combined percentage error for the control population was $17.2 \pm 2.0(n=30)$ and for experimentals it was $43.1 \pm 7.41(n=10 ; t=-3.99 ; p<0.01)$. Taken together, these results suggest reshaping of contributions to recorded activity across the entire bouton ensemble (see more details in Discussion).

\section{Bouton size and density}

Microscopic examination of the terminals of these fibers suggested that following regeneration many fibers supported fewer boutons and that these remaining boutons were smaller than those observed in controls. In order to assess the validity of these subjective observations, boutons were counted and measured for both experimental and control fibers and compared directly. To avoid any possible bias due to differences in the quality of staining between control and experimental fibers and/ or differences due to laminar location (i.e., control fibers were all cutaneous fibers), we chose only "originally cutaneous" experimental fibers in which stained boutons could be observed over at least $6 \mathrm{~mm}$ within laminae III-V. A total of five regenerated afferents met these criteria and were used for the comparisons. As a further safeguard only the $3 \mathrm{~mm}$ of continuous dorsal horn containing the greatest number of boutons were used for the comparisons. These counts revealed that the regenerated fibers supported significantly fewer boutons per millimeter $(932 \pm 202.17 ; n=5)$ in the dorsal horn than did control 


\section{cutaneous to skin}

A

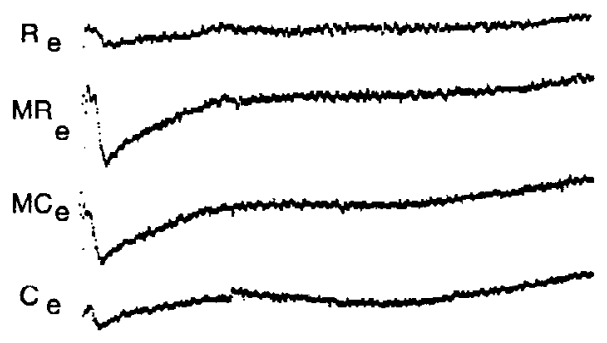

B

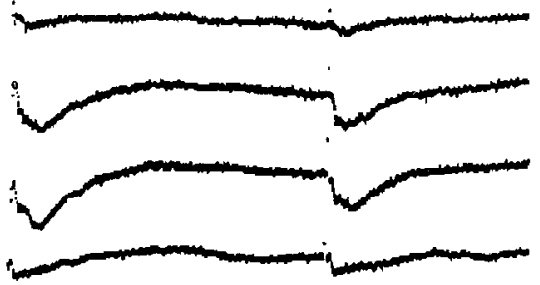

\section{cutaneous to skin}

C

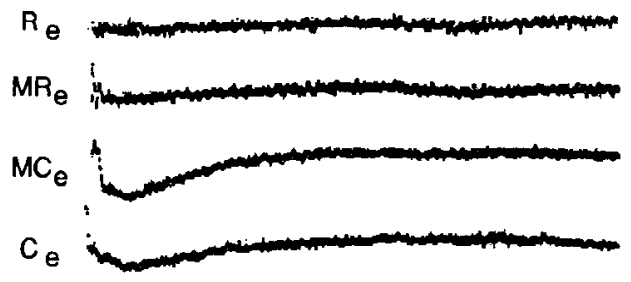

D

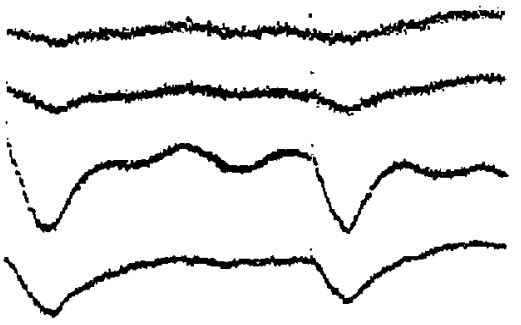

\section{cutaneous to subcutaneous}

$E$

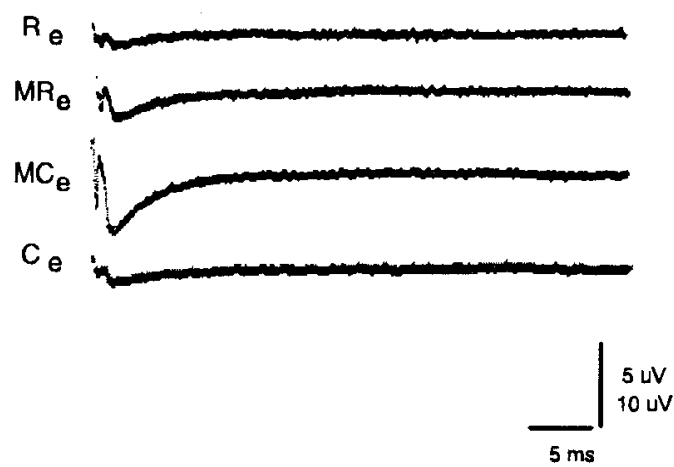

$F$

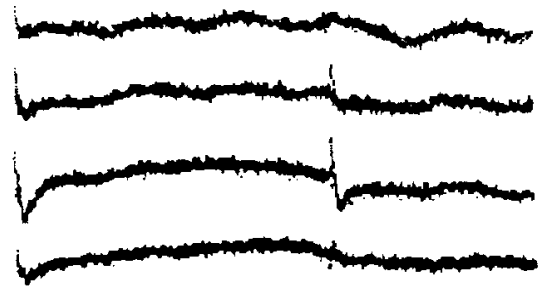
$\left.\right|_{10 \mathrm{~ms}} 10 \mathrm{uv}$
Figure 9. $A, C$, and $E, C D P s$ evoked in response to $18 \mathrm{~Hz}$ of three different $\mathrm{A} \beta$-fibers judged to have innervated cutaneous LTMRs before axotomy. $B, D$, and $F$, CDPs evoked in response to pairs of shocks (ISI $=50 \mathrm{msec}$ ) delivered every $2 \mathrm{sec}$ to the same afferents. Amplitude calibration: $A$ and $C, 5 \mu \mathrm{V}, B$ and $D-F, 10 \mu \mathrm{V}$. fibers $(1560 \pm 129.09 ; n=17 ; t=2.28 ; p<0.05$; summarized in Table 2).

A similar selection process was necessary to choose the appropriate fibers for measuring the arcas of boutons. Since the control population consisted of cutaneous LTMR fibers, the size measurements were restricted to the boutons of regenerated cutaneous fibers in laminae III-V. There were six regenerated afferent fibers that met these requirements. We included one afferent (afferent 13, Table 1) that was classified as "sprouted cutaneous" in this group because of the level of staining and the large numbers of boutons in laminae III and IV (Fig. 11A). An average of 267 boutons were measured for each afferent fiber (range of 249-289). It was found that regenerated afferents supported significantly smaller boutons $\left(1.76 \pm 0.16 \mu \mathrm{m}^{2} ; n=6\right)$ than those observed for intact fibers $\left(2.23 \pm 0.08 \mu \mathrm{m}^{2} ; n=8\right.$; ANOVA, $p=0.03$ ). As stated in Materials and Mcthods these measurements were computer assisted and by the nature of the process (e.g., the program determining the edges of the stained material) the actual bouton area may be somewhat underestimated. However, as this was designed as a "blind" procedure, any such errors should cancel out.

These results suggest that following regeneration afferent fibers supported fewer boutons, of smaller size, than they did prior to axotomy. However, as pointed out above, these fibers 
evoked CDPs with amplitudes equal to or greater than those evoked by intact afferents. A further quantification of these rcsults was obtained by computing the average contribution of each bouton (amplitude coefficient, $a$ ) to the evoked monosynaptic CDPs as was done for intact fibers in an earlier investigation (Koerber et al., 1990). These calculations were limited to results from the same five afferents selected above and revealed that the average contribution per bouton had increased by greater than threefold (control $a=0.14 \pm 0.02 \mu \mathrm{V} ; n=16$ ), experimental $a=0.46 \pm 0.18 \mu \mathrm{V} ; n=5 ; t--3.09 ; p<0.01$ ). These findings confirm an increase in the contribution by individual boutons to the potential recorded.

One possible reason for this apparent increase in synaptic efficacy would be a general change in the volume conductor characteristics of the spinal cord in response to the axotomy and regeneration process. To control for this possibility we recorded CDPs evoked by stimulation of intact peroneal afferents in the experimental animals and compared the results to those for peroneal afferents recorded in the previous studies in normal animals. Although tibial and peroneal fibers project to separate mediolateral locations in the dorsal horn they overlap extensively in the rostrocaudal axis and therefore any general change in the volume conductor would probably affect the amplitude of CDPs evoked by these afferents. The results from this comparison are summarized in Table 2 . The CDPs evoked by stimulation of intact peroneal afferents in experimental animals were found to be of the same amplitude as those evoked by peroneal afferents in normal animals. Therefore, it is unlikely that a nonspecific change in the volume conductor is responsible for the increase in the amplitude coefficient observed for the regenerated tibial fibers.

\section{Discussion}

This series of experiments was designed to evaluate the effects of axotomy and the ensuing reinnervation of peripheral targets on sensory information processing. Since regenerating fibers can reinnervate novel tissue and/or receptors it is necessary to have some means of determining the appropriateness of reinnerva-

\section{cutaneous to skin}

A

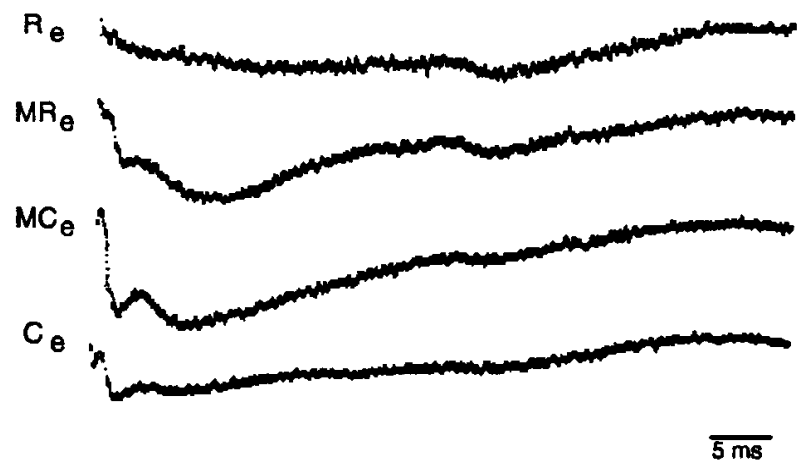

B

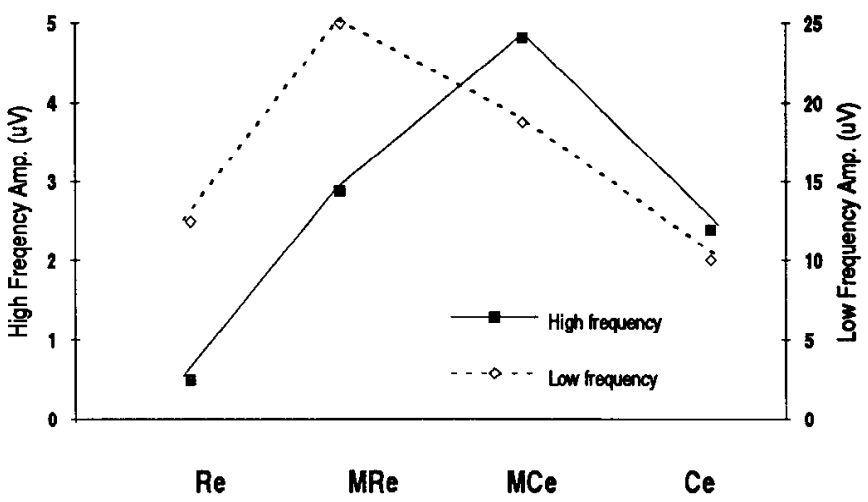

Figure 10. $A, \mathrm{CDPs}$ recorded in response to $18 \mathrm{~Hz}$ stimulation of $\mathrm{A} \beta$ fiber depicted in Figure $8 B$. Note the slow rise time polysynaptic component to the CDP. $B$, Plots of the peak amplitude of the CDPs recorded at the four site in response to $18 \mathrm{~Hz}$ (solid squares) and $0.5 \mathrm{~Hz}$ (open diamonds) stimulation. Note that the largest response to the different frequencies of stimulation are not at the same rostrocaudal location.

Table 2. Summary of measurements for selected regenerated and intact afferent fibers

\begin{tabular}{|c|c|c|c|c|c|}
\hline & $\begin{array}{l}\text { Longitudinal } \\
\text { density } \\
\text { (btns/mm) }\end{array}$ & $\begin{array}{l}\text { Bouton size } \\
\left(\mu \mathrm{m}^{2}\right)\end{array}$ & $\begin{array}{l}\text { Peak amplitude } \\
(\mu \mathrm{V})\end{array}$ & $\begin{array}{l}\text { Amplitude } \\
\text { coefficient } \\
(\mu \mathrm{V})\end{array}$ & \% Error \\
\hline \multicolumn{6}{|l|}{ Intact tibial } \\
\hline $\begin{array}{l}\text { afferents in } \\
\text { controls }\end{array}$ & $\begin{array}{l}1560 \pm 129 \\
(n=17)\end{array}$ & $\begin{array}{l}2.23 \pm 0.08 \\
(n=8)\end{array}$ & $\begin{array}{l}3.55 \pm 0.74 \\
(n=8)\end{array}$ & $\begin{array}{l}0.14 \pm 0.02 \\
(n=15)\end{array}$ & $\begin{array}{l}17.2 \pm 2.0 \\
(n=30)\end{array}$ \\
\hline $\begin{array}{l}\text { Regenerated } \\
\text { tibial afferents }\end{array}$ & $\begin{array}{l}932 \pm 202 * \\
(n=5)\end{array}$ & $\begin{array}{l}1.76 \pm 0.16^{* *} \\
(n=6)\end{array}$ & $\begin{array}{l}5.16 \pm 0.89 \\
(n=5) \\
\text { RAs: } 2.27 \pm 0.28\end{array}$ & $\begin{array}{l}0.55 \pm 0.18^{*} \\
(n=5)\end{array}$ & $\begin{array}{l}43.1 \pm 7.1^{*} \\
(n=10)\end{array}$ \\
\hline $\begin{array}{l}\text { Intact peroneal } \\
\text { afferents in } \\
\text { controls }\end{array}$ & - & - & $\begin{aligned} & (n=20) \\
\text { SAs: } & 3.52 \pm 0.38 \\
& (n=11)\end{aligned}$ & - & - \\
\hline $\begin{array}{l}\text { Intact peroneal } \\
\text { afferents in } \\
\text { experimentals }\end{array}$ & - & - & $\begin{aligned} \text { RAs: } & 1.83 \pm 0.26 \\
& (n=12) \\
\text { SAs: } & 3.04 \pm 0.45 \\
& (n=15)\end{aligned}$ & - & - \\
\hline
\end{tabular}

Data are presented as mean \pm SEM

${ }^{*} p<0.05$, two-tailed Student $t$ test.

${ }^{* *} p<0.05$ ANOVA. 


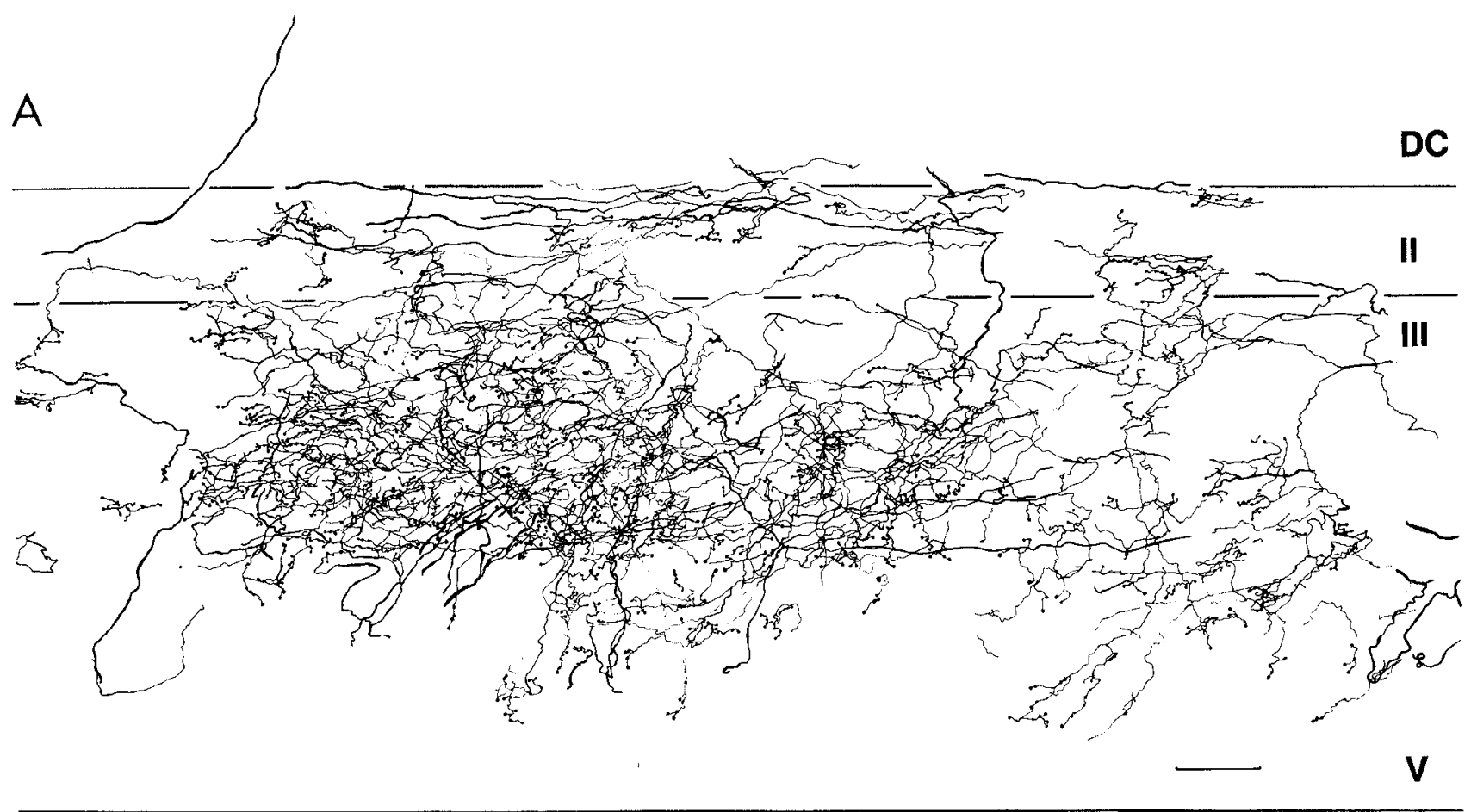

B

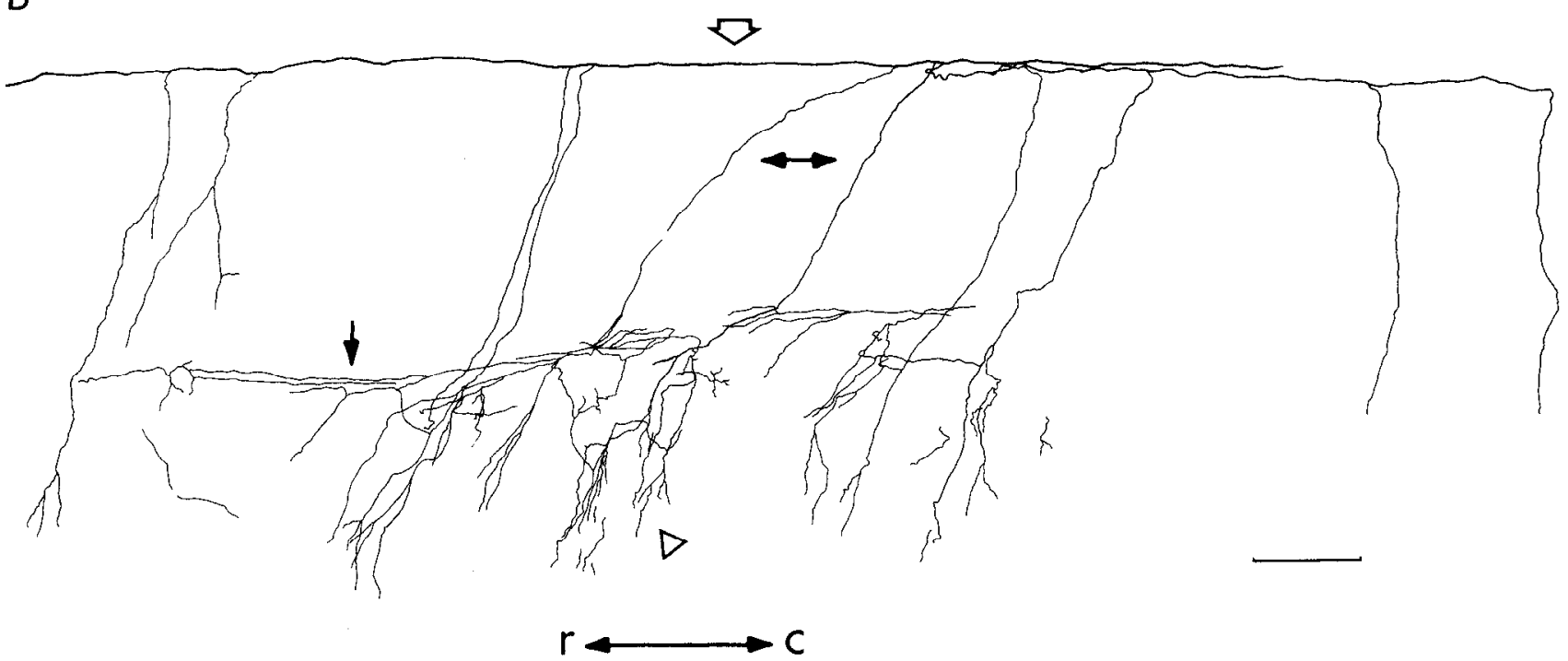

Figure 11. A, Camera lucida drawing of a portion of the central projections of an A $\beta$-fiber $(\mathrm{CV}=69 \mathrm{~m} / \mathrm{sec})$. This fiber was classified as a sprouted cutaneous fiber due to the projections located in the superficial laminae. CDPs evoked by stimulation of this afferent can be seen in Figure $12, A$ and $B$. Note the density of boutons in laminae III-IV. $B$, Camera lucida drawing of the central projections of a second $A \beta$-fiber with boutons located in laminae I-V. This low-magnification drawing depicts the main collateral in the dorsal columns (open arrow), the descending collaterals (double solid arrow), the longitudinally oriented fibers in the superficial laminae (single solid arrow) and the transversely oriented fibers in the nucleus proprius (open triangle). $r$, rostral; $c$, caudal. Scale bars: $A, 100 \mu \mathrm{m} ; B, 350 \mu \mathrm{m}$.

tion at the single fiber level. In the present study the individual fibers were stained and their central projections reconstructed so that the preaxotomy identity of the afferents could be determined by the laminar locations of their spinal projections. The central projections of most of the afferents presented in this study (10 of 13) were qualitatively indistinguishable from those of controls. In addition the other three afferents had projections confined to laminae I-V, making general classification (i.e., cutaneous vs proprioceptive) straightforward. Additional information such as conduction velocity and specific termination patterns permitted more precise identification (e.g., Ia muscle spindle). This approach allowed us to address both questions of specificity of regeneration as well as the consequences of inappropriate reinnervation. 
sprouted cutaneous to subcut. LTMR

A
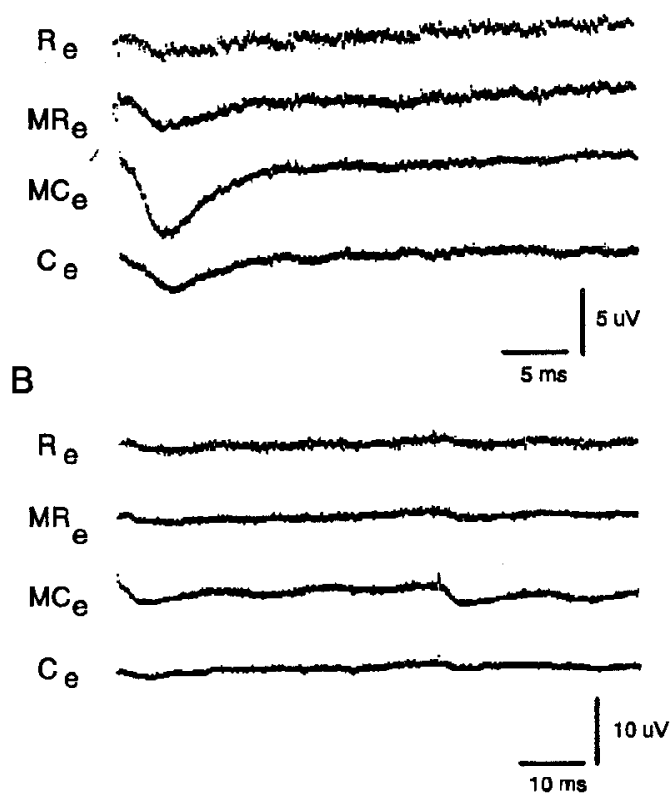

sprouted cutaneous to cut. LTMR

C

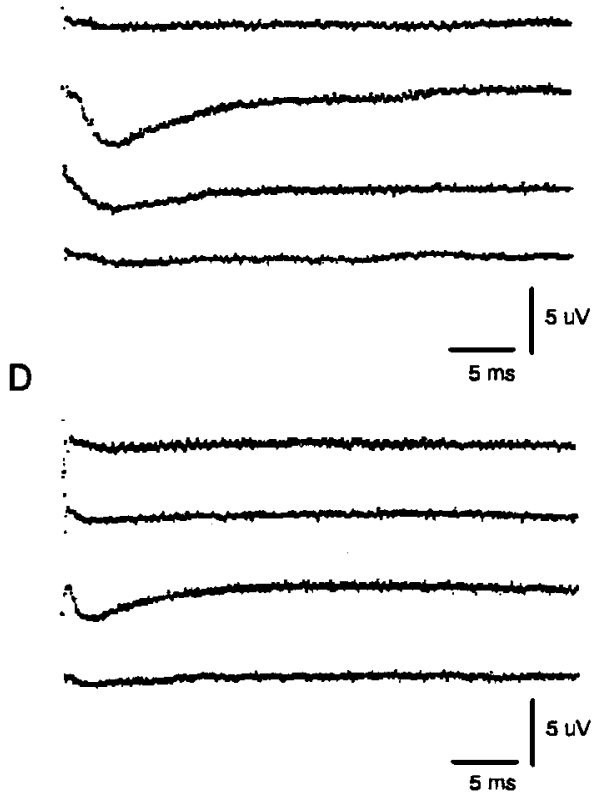

Figure 12. CDPs evoked by stimulation of sprouted cutaneous fibers. $A$, CDPs evoked by $18 \mathrm{~Hz}$ stimulation of the fiber in Figure $11 A . B$, CDPs evoked by the same fiber in response to pairs of shocks (ISI $=50 \mathrm{msec}$ ) delivered every 2 sec. $C$, CDPs evoked by $18 \mathrm{~Hz}$ stimulation of a second $\mathrm{A} \beta$-fiber $(\mathrm{CV}=$ $62 \mathrm{~m} / \mathrm{sec}$ ). $D$, CDPs evoked by $18 \mathrm{~Hz}$ stimulation of the third $\mathrm{A} \beta$-fiber $(\mathrm{CV}=$ $68 \mathrm{~m} / \mathrm{sec}$ ) supporting collaterals in the superficial dorsal horn. Note the slow rise time of the CDPs in response to 18 $\mathrm{Hz}$ stimulation.

\section{Proprioceptive fibers}

Although anatomical characterization of proprioceptive afferents was very straightforward, their physiological characteristics were not consistent with the normal population. Unlike control proprioceptive fibers many of these regenerated axons evoked measurable monosynaptic CDPs in response to $18 \mathrm{~Hz}$ stimulation. Comparison of the central projections of regenerated proprioceptive fibers producing CDPs with those that did not revealed differences in the relative laminar distribution of boutons. Fibers evoking CDPs supported many more dorsally located (laminae IV-V) boutons than those that did not. This was true for fibers in both the group I and II conduction velocity range. Initially it was thought this correlation might indicate that axotomy induces increased numbers of dorsally located boutons for selected fibers (e.g., group I muscle spindle afferent with many boutons in laminae IV and V). However, careful examination of earlier publications revealed that the central projections of these fibers were well within the range of projections reported for normal proprioceptive fibers (Ishizuka et al., 1979; Brown, 1981; Mense, 1990). Thus, the functional change observed in response to axotomy need not he mediated by the establishment of new synapses in more dorsally located regions of the spinal cord. The most parsimonious explanation would be that those afferents that normally have more dorsally located boutons can now produce measurable CDPs because of an increase in synaptic efficacy of these boutons. That is, the increased synaptic efficacy could be the same for connections in laminae IV and V as in laminae VI and VII and the reason that those in IV and $V$ produce measurable CDPs is their closer proximity to the cord surface. However, recent studies have shown that large-diameter cutaneous afferents are capable of sprouting into denervated regions of the dorsal horn (Molander et al., 1988; LaMotte and Kapadia, 1993). Since synapse elimination has also been suggested as the stimulus for sprouting of large-caliber fibers into the substantia gelatinosa (Woolf et al., 1992), the decrease in bouton densities reported here would suggest that the same stimulus is present (albeit to a lesser degree) in the nucleus proprius. Therefore, these results are also consistent with the hypothesis that some proprioceptive fibers had undergone collateral sprouting and are now supporting more dorsally located boutons that have formed new functional connections in the dorsal horn.

Regardless of the mechanism, these fibers do exhibit a striking functional change. These findings are also in accord with the results of the recent studies of Lewin and McMahon (1991c), in which they demonstrated an increase in the responsiveness of dorsal horn cells to muscle afferent inputs after the afferents had been rerouted to reinnervate skin. From their results and those of earlier studies (McMahon and Gibson, 1987; McMahon and Moore, 1988; McMahon et al., 1989), these authors suggested that the muscle afferents have changed their central connectivity in response to the reinnervation of inappropriate target tissue, thus becoming more like cutaneous afferents. However, our results suggest that given the right stimulus this functional change may occur even if the proprioceptive afferents return to muscle (e.g., Fig. 3). Furthermore, since the results of this study show changes in the monosynaptic CDPs generated by the proprioceptive afferents, we can conclude that at least some of the reported changes in dorsal horn excitability are occurring in cells receiving direct input from primary afferents. However, it cannot be determined whether these changes are due to establishment of new connections or modification of existing ones.

These monosynaptic CDPs were similar to cutaneous fiberevoked CDPs in onset latency and rise time, but were generally smaller in amplitude. As noted earlier the CDPs recorded at the different sites did not show the same variations in amplitude even though the longitudinal distributions of boutons were very similar to those seen in controls. Thus, the correlation between the bouton distribution and amplitude profile is not as good for 
A cutaneous to skin

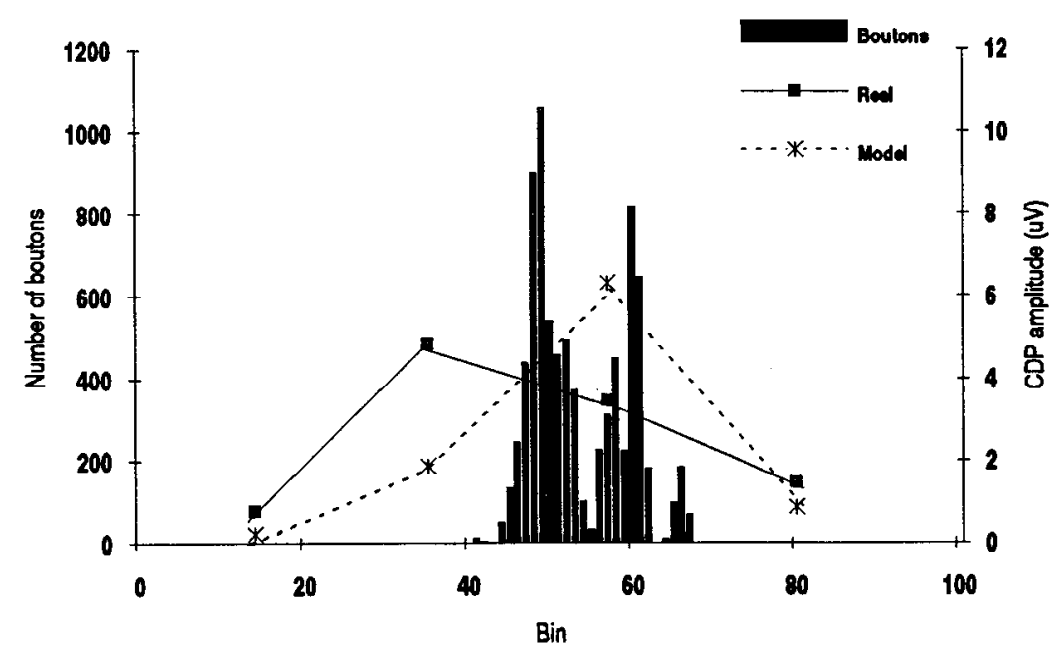

B cutaneous to skin

C cutaneous to skin

Figure 13. $A-E$, Plots for five cutaneous fibers of the longitudinal distribution of boutons ( $250 \mu \mathrm{m}$ bins), the amplitudes of the CDPs recorded at each site in response to $18 \mathrm{~Hz}$ stimulation, and the amplitudes predicted using the calculated amplitude coefficient $a$ and the bouton distributions.
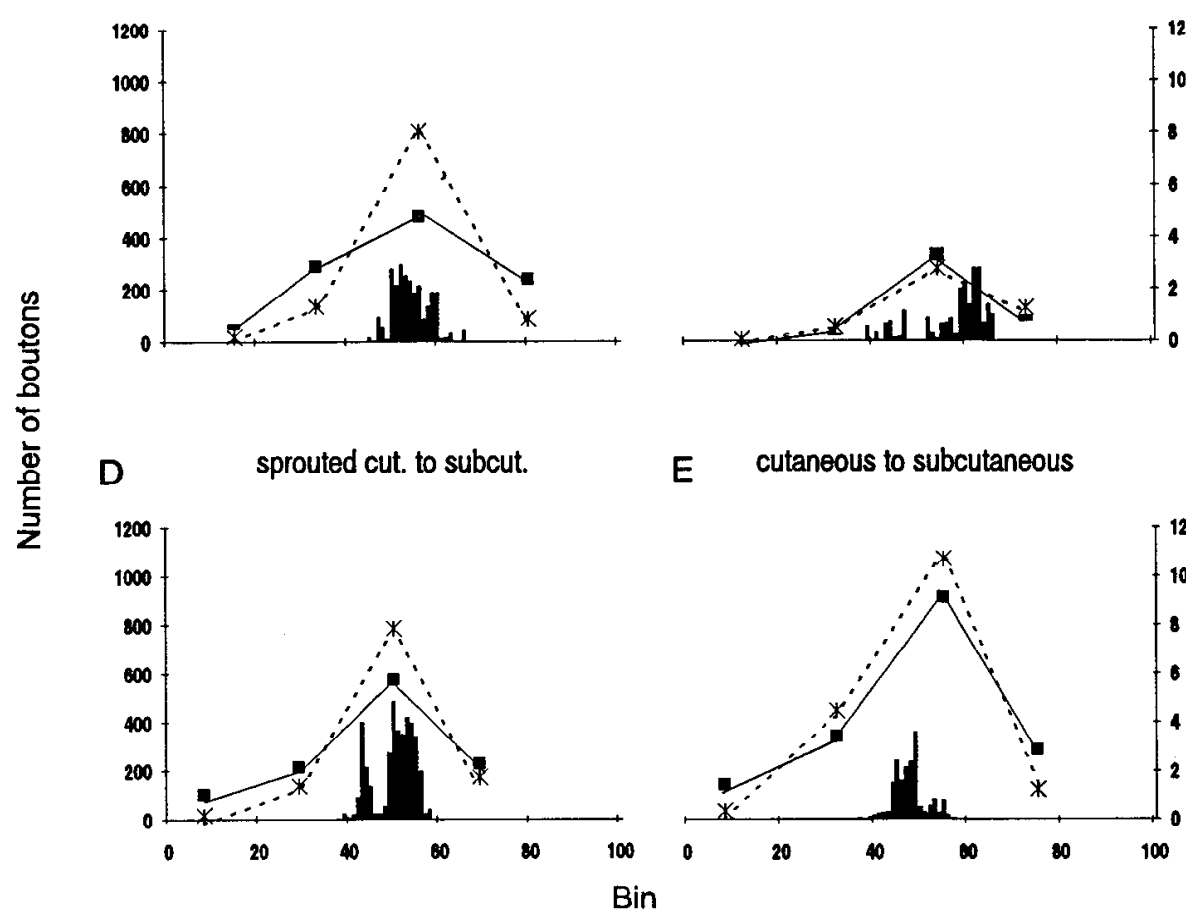

these fibers as it is for control cutaneous fibers. This could be due to the fact that proprioceptive afferents contact a different subset of dorsal horn cells that have significantly different dendritic geometry. Furthermore, unlike the cutaneous fibers in this study and those from controls (Kocrber and Mendell, 1988) the regenerated proprioceptive fibers that evoked measurable monosynaptic CDPs did not evoke CDPs of greater amplitude when the stimulus rate was reduced. This would also suggest that the proprioceptive afferents are projecting to a subset of dorsal horn cells that do not activate cutaneous afferent-driven networks, which produce CDPs of greater amplitude as stimulus frequency is reduced (Koerber and Mendell, 1988; Koerber et al., 1991).

\section{Cutaneous afferents}

Those regenerated fibers considered originally to have been cutaneous afferents on the basis of their central projections were divided into two groups based on the presence or absence of collaterals in laminae I and II. The fibers with projections confined to laminae III-V were indistinguishable from intact controls. In fact, many of these could be classified further as specific types of cutaneous LTMRs. An example of this homology can be seen by comparing the afferent projections depicted in Figure $8 B$ with the projections of the slowly adapting type I afferent depicted in Figure $2 A$ of Koerber et al. (1990). However, this afferent exhibited a rapidly adapting response to mechanical 
stimulation of the skin and, as seen normally for fibers innervating $\mathrm{RA}$ receptors, considerable depression of the test CDP in response to the pairs of shocks. The depression was greater than that seen for SAs in controls. Nonetheless, the configuration of the CDPs more closely resembled those evoked by intact SA afferents than RA afferents. Actually, this afferent evoked polysynaptic CDP components even at high-frequency stimulation (Fig. 104), suggesting changes in synaptic efficacy at other connections in the single afferent-driven networks. The other previously cutaneous afferents evoked CDPs that were consistent with their central anatomy and peripheral response type.

Regenerated fibers whose central projections included extensive collateralization in more superficial laminae (I and II) were the only fibers whose central terminal distributions could be considered atypical. In an earlier preliminary publication (Koerber et al., 1989), one of four stained fibers described exhibited this distribution of central terminals. This fiber had reinnervated the central foot pad and responded to low-threshold stimuli in a rapidly adapting manner. It was concluded that in this case we had stained a fiber that had originally been an A $\beta$ HTMR and that it had reinnervated a cutaneous LTMR. This conclusion was based largely on the absence of any evidence for the alternative explanation, that an afferent previously innervating a cutaneous LTMR had reinnervated a cutaneous LTMR but had also sprouted into superficial laminae and maintained these sprouts for more than a year.

However, with the additional two afferents reported here along with the recent results of Woolf et al. (1992) and Shortland and Woolf (1993), we should reevaluate this conclusion. Taken together, these results now strongly suggest that the central projections of some $A \beta$-fibers previously innervating LTMRs sprout into novel dorsal horn laminae in response to peripheral axotomy.

The fact that these fibers evoke monosynaptic CDPs that are similar in configuration to those produced by identified nociceptors in controls and that they exhibit facilitation in response to the conditioning-testing pairs paradigm strongly suggests that these dorsal sprouts make functional connections in these laminae. It should also be noted that the monosynaptic CDPs evoked by these afferents in response to $18 \mathrm{~Hz}$ stimulation are much larger in amplitude $(3.84 \pm 1.18 \mu \mathrm{V})$ than those observed for nociceptors in the control population that rarely measured more than $1 \mu \mathrm{V}$. Therefore, it is most likely that CDPs evoked by stimulation of these afferents are the result of activity at the connections in all the laminae containing boutons.

In summary, qualitative evaluation of the anatomy and physiology of these regenerated afferents provided two lines of evidence for plasticity of the central terminals of regenerated afferents. First, the connections between dorsal horn cells and fibers judged to have been proprioceptors previously have undergone modifications following axotomy such that they became capable of evoking measurable CDPs. As pointed out previously this does not appear to be the result of a general change in the volume conductor in the dorsal horn because intact afferents in the experimental preparations evoked the same size CDPs as in controls. Second, some cutaneous afferents sprouted into laminae I and II and made functional connections with cells in these laminae. The exact stimulus for this sprouting is difficult to ascertain. As mentioned above, Woolf et al. (1992) suggested that the sprouting may be induced by the degeneration of the terminations of small-caliber fibers in the substantia gelatinosa. Results from the present study do not suggest any other potential stimuli for the observed sprouting. However, we can add that these sprouts apparently make functional connections which persist long after successful reinnervation of the appropriate peripheral targets (i.e., cutaneous LTMR to cutaneous LTMR).

Another interesting observation that addresses a mechanism of the observed plasticity and is consistent across both studies is that the sprouting fibers appear to maintain the same mediolateral position in the dorsal horn as the parent collaterals. In the present study the sprouted fibers penetrated the superficial laminae and turned to run longitudinally sometimes for several hundreds of micrometers. However, they always remained in the same relative mediolateral position as those in the deeper projections. This suggests that although the process of axotomy/ regeneration triggers a central mechanism to induce or allow growth of fibers to novel locations, this sprouting of selected fibers appears to be directed in some manner relative to the somatotopic organization of the dorsal horn.

\section{Specificity of regeneration}

The regenerated afferents reported here exhibit very little specificity in their choice of peripheral targets. Among the six previously proprioceptive fibers, five reinnervaled the skin, and two of the seven previously cutaneous afferent fibers reinnervated subcutaneous structures. These results are in agreement with previous investigations that document the apparent inability of regenerating afferent fibers to choose the appropriate peripheral conduit to return them to their original target type (Brushart, 1988; Koerber et al., 1989). However, the actual percentages in this study (i.e., $>80 \%$ of proprioceptors reinnervating the skin) may be misleading. Since only one afferent could be injected per experiment, precise evaluation of the peripheral response properties was critical. Fibers reinnervating the skin were more accessible for the necessary manipulations; thus, there is a sampling bias toward those fibers.

One property of the afferents' function that exhibited a high degree of homology with their central projection patterns following regeneration was their peripheral adaptation properties. For the previously proprioceptive fibers five of six were classified as slowly adapting. Likewise, three of four cutaneous fibers also exhibited the appropriate match between peripheral adaptation properties and their central projection patterns and function. These results are also in agreement with previous studies (Lewin and McMahon, 1991a; Johnson and Munson, 1992) demonstrating that when muscle afferents were forced to reinnervate the skin there was a strong tendency for them to remain slowly adapting. This tendency for regenerated fibers to retain their peripheral adaptive properties was also reported for cutaneous afferents returning to the skin (Burgess and Horch, 1973). Thus, the majority of regenerated afferent fibers retain their original peripheral adaptation properties. However, it is not clear whether this is due to their locating the correct receptor type or whether it occurs in spite of the receptor type reinnervated. For example, it has been shown that in the absence of receptors, axotomized muscle afferents can exhibit relatively normal rcsponsc properties (Johnson and Munson, 1991). Therefore, it is possible that the observation in the present study may reflect the retention of intrinsic membrane properties of individual afferents fibers rather than specificity of regeneration.

\section{Anatomical and physiological correlates}

The availability of both anatomical and physiological data for the same afferents allowed for a more complete evaluation of 
the results, which revealed other changes induced by the axotomy/regeneration process. For example, quantitative examination of these regenerated cutaneous afferents revealed that they had fewer and smaller synaptic boutons than intact cutaneous afferents. However, the monosynaptic CDPs evoked by these afferents were on average the same or larger than those measured in controls. Therefore, whatever the mechanism responsible for the observed decreases in bouton size and number, there was a compensatory mechanism that apparently increased the average synaptic efficacy of the remaining connections. As pointed out earlier, these changes could be presynaptic (e.g., increase in efficacy of spike invasion of terminal branches, postsynaptic (e.g., changes in membrane resistance), or both (e.g., increases in numbers or sizes of synaptic specializations per bouton). Another possible mechanism is a generalized loss of inhibition (e.g., a loss of primary afferent depolarization; Horch and Lisney, $1981 \mathrm{~b}$ ).

This increase in synaptic efficacy could be explained by a general phenomenon induced by the axotomy/regeneration process that was restricted to axotomized afferent connections. Likewise, the computed amplitude coefficients $a$ for the regenerated afferents, which were significantly larger, could be due to such a general effect. However, when this coefficient was used along with the longitudinal distribution of boutons to calculate the expected amplitude of the CDPs at each recording site and these values compared with the observed potentials, there was a 2.5-fold increase in the percentage error of expected versus observed CDP amplitudes compared to controls. This suggests a basic change in the way connections within different parts of the rostrocaudal distribution of boutons contribute to the observed potential. One possible explanation for these findings is that the contribution per bouton is independent of longitudinal position in controls, and nonuniformly distributed following axotomy and regeneration.

This possibility is consistent with other results suggesting that dorsal horn cells do not maintain the same level of connectivity with a given fiber following regeneration because of the scrambling effects of peripheral axotomy on somatotopy. That is, although regenerating axons select distal Schwann cell conduits in a relatively random manner and therefore seldom return to their appropriate peripheral locations (Burgess and Horch, 1973; Horch, 1979; Horch and Lisney, 1981), over time dorsal horn cells are capable of reconstructing relatively concise cutaneous receptive fields from these scrambled inputs (Lisney, 1983). More recently, results from McMahon and Lewin (1992) and Koerber and Mirnics (1993) suggest that shortly after successful reinnervation dorsal horn cells receiving input from the regenerated afferents have very large receptive fields and that with time these low-threshold receptive fields shrink back to more normal size. Thus, the regional changes in the efficacy of groups of boutons observed for the single afferents in this study could be the result of this restructuring of dorsal horn cell receptive fields.

\section{Summary and conclusion}

The present findings help us elucidate the effects of peripheral axotomy and regeneration on sensory information processing in the dorsal horn. We have confirmed earlier findings that this process results in mismatches between the peripheral response properties and central anatomy and function. These mismatches obviously have profound effects on information processing. However, at the single-fiber level the effects of faulty reinner- vation are more subtle as most of the findings in this study could be viewed as general effects of axotomy and regeneration. For example, proprioceptive afferents can exhibit changes centrally (functional or anatomical and functional) even though they return to muscle. Likewise, large-diameter cutaneous afferents that return to the skin exhibit anatomical and functional changes. Thus, for large-diameter afferents specificity of reinnervation is not necessarily a critical factor in the initiation of central plastic events.

\section{References}

Adams JC (1977) Technical considerations on the use of horseradish peroxidase as a neuronal marker. Neuroscience 2:141-145.

Banks RW, Barker D, Stacey MJ (1984) Reinnervation of cat muscle spindles by foreign afferents after nerve section. J Physiol (Lond) 357: $21 \mathrm{P}$.

Brown AG (1981) Organization in the spinal cord. New York: Springer.

Brushart TME (1988) Preferential reinnervation of motor nerves by regenerating motor axons. J Neurosci 8:1026-1031.

Burgess PR, Horch KW (1973) Specific regeneration of cutaneous fibers in the cat. J Neurophysiol 36:101-114.

Ford FR, Woodhall B (1938) Phenomena due to misdirection of regenerating fibers of cranial spinal and autonomic nerves. Arch Surg $36: 480-499$.

Hawkins GL (1948) Faulty localization in nerve regeneration. An index of functional recovery following suture. J Neurosurg 5:11-18.

Hoffer JA, Stein RB, Gordon T (1979) Differential atrophy of sensory and motor fibers following section of cat peripheral nerves. Brain Res 178:346-361

Horch KW (1979) Guidance of regrowing sensory axons after cutaneous nerve lesions in the cat. J Neurophysiol 42:1437-1499.

Horch KW, Lisney SJW (1981a) On the number and nature of regenerating myelinated axons after lesions of cutaneous nerves in the cat. J Physiol (Lond) 313:275-286.

Horch KW, Lisney SJW (1981b) Changes in primary afferent depolarization of sensory neurons during peripheral nerve regeneration the cat. J Physiol (Lond) 313:287-299.

Ishizuka N, Mannen H, Hongo T, Sasaki S (1979) Trajectory of group Ia afferent fibers stained with horseradish peroxidase in the lumbosacral spinal cord of the cat: three dimensional reconstructions from serial sections. J Comp Neurol 186:189-211.

Johnson RD, Munson JB (1991) Regenerating sprouts of axotomized cat muscle afferents express characteristic firing patterns to mechanical stimulation. J Neurophysiol 66:2155-2158.

Johnson RD, Munson JB (1992) Specificity of regenerating sensory neurons in adult mammals. In: Sensory neurons: diversity, development and plasticity (Scott SA, ed). New York: Oxford UP.

Koerber HR, Mendell LM (1988) Functional specialization of central projections from identified primary afferent fibers. J Neurophysiol 60:1597-1614.

Koerber HR, Mirnics K (1993) Plasticity of dorsal horn cell receptive fields following peripheral nerve regeneration. Soc Neurosci Abstr 19: 1563 .

Koerber HR, Druzinsky RE, Mendell LM (1988) Properties of somata of spinal dorsal root ganglion cells. J Neurophysiol 60:1584-1596.

Koerber HR, Seymour AW, Mendell LM (1989) Mismatches between peripheral receptor type and central. Neurosci Lett 99:67-72.

Koerber HR, Brown PB, Mendell LM (1990) Correlation of monosynaptic field potentials evoked by single action potentials in single primary afferent axons and their bouton distributions in the dorsal horn. J Comp Neurol 294:133-144.

Koerber HR, Seymour AW, Mendell LM (1991) Tuning of spinal networks to frequency components of spike. J Neurosci 11:31783187 .

Koerber HR, Mirnics K, Brown PB, Mendell LM (1992) Sprouting and functional plasticity of spinal projections of regenerated primary afferents. Soc Neurosci Abstr 18:133.

LaMotte CC, Kapadia SE (1993) Deafferentation-induced terminal field expansion of myelinated saphenous afferents in the adult rat dorsal horn and the nucleus gracilis following pronase injection of the sciatic nerve. J Comp Neurol 330:83-94.

Lewin GR, McMahon SB (1991a) Physiological properties of primary 
afferents appropriately and inappropriately innervating skin in adult rats. J Neurophysiol 66:1205-1217.

Lewin GR, McMahon SB (1991b) Physiological properties of primary afferents appropriately and inappropriately innervating skeletal muscle in adult rats. J Neurophysiol 66:1218-1231.

Lewin GR, McMahon SB (1991r) Reorganization of primary afferent connectivity in the adult spinal cord: evidence for tissue-specific neurotrophic influences from the periphery. Eur J Neurosci 3:1 112-1122.

Lindblom U, Verillo RT (1979) Sensory functions in chronic neuralgia. J Neurol Neurosurg Psychiatry 42:422-435.

Lisney SJW (1983) Changes in somatotopic organization of the cat lumbar spinal cord following peripheral nerve transection and regeneration. Brain Res 259:31-39.

McMahon SB, Gibson S (1987) Peptide expression is altered when afterent nerves reinnervate inappropriate tissue. Neurosci Lett 73:915.

McMahon SB, Lewin GR (1992) NMDA receptor mediated plasticity in the dorsal horn after peripheral nerve injury. Soc Neurosci Abstr 18:443.

McMahon SB, Moore CEG (1988) Plasticity of primary afferent acid phosphatase expression following rerouting of afferents from muscle to skin in the adult rat. J Comp Neurol 274:1-9.

McMahon SB, Lewin GR, Anand P, Ghatei MA, Bloom SR (1989)
Quantitative analysis of peptide levels and neurogenic extravasation following regeneration of afferents to appropriate and inappropriate targets. Neuroscience 33:67-75.

Mense S (1990) Structure-function relationships in identified afferent neurones. Anat Embryol (Berl) 181:1-17.

Moberg E (1962) Criticism and study of methods for examining sensibility in the hand. Neurology 12:8-29.

Molander CE, Kinnman E, Aldskogius H (1988) Expansion of spinal cord primary sensory afferent projection following combined sciatic nerve resection and saphenous nerve crush: a horseradish peroxidase study in the adult rat. J Comp Neurol 276:436-441.

Nishimura H, Johnson RD, Munson JB (1993) Rescue of ncuronal function by cross-regeneration of cutaneous afferents into muscle in cats. J Neurophysiol, in press.

Onne L (1962) Recovery of sensibility and sudomotor activity in the hand after nerve suture. Acta Chir Scand [Suppl] 300:1-69.

Shortland P, Woolf CJ (1993) Chronic peripheral nerve section results in a rearrangement of the central axonal arborizations of axotomized A-beta primary afferent neurons in the rat spinal cord. J Comp Neurol 330:65-82.

Woolf CJ, Shortland P, Coggeshall RE (1992) Peripheral nerve injury triggers central sprouting of myelinated afferents. Nature 355:75-78. 